\title{
"Present and future of immunotherapy in Neuroendocrine Tumors"
}

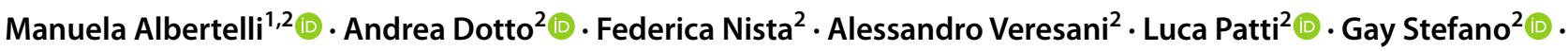 \\ Stefania Sciallero ${ }^{3}(\mathbb{D}) \cdot$ Mara Boschetti $^{1,2}$ (D) Diego Ferone $^{1,2}$ (])
}

Accepted: 16 March 2021 / Published online: 14 April 2021

(c) The Author(s) 2021

\begin{abstract}
Immunotherapy, so promising in many neoplasms, still does not have a precise role in the treatment of neuroendocrine neoplasms (NENs). In this article, we provide an overview on the current knowledge about immunotherapy with immune checkpoint inhibitors (ICIs) applied to NENs, evaluating future perspectives in this setting of tumors.

Evidence so far available for ICIs in gastroenteropancreatic (GEP)-NENs is definitively not as robust as for other tumors such as Small Cell Lung Cancer or Merkel Cell Carcinoma. In fact, with regard to the well-differentiated forms of NENs (NETs), the results obtained nowadays have been disappointing. However, the near future, might reserve interesting results for ICIs in GEP-NEN from a total of nine different ICI drugs, used throughout 19 randomised controlled trials. Such numbers highlight the growing attention gathering around NENs and ICIs, in response to the need of stronger evidences supporting such therapy. For the future, the most important aspect will be to study strategies that can make NETs more susceptible to response to ICI and, thus, enhance the effectiveness of these treatments. Therefore, the combination of conventional therapy, target therapy and immunotherapy deserve attention and warrant to be explored. A sequential chemotherapy, possibly inducing an increase in tumor mutational burden and tested before immunotherapy, could be a hypothesis deserving more consideration. A radiation treatment that increases tumor-infiltrating lymphocytes, could be another approach to explore before ICIs in NENs. Equally essential will be the identification of biomarkers useful for selecting patients potentially responsive to this type of treatment.
\end{abstract}

Keywords Immunotherapy $\cdot$ Immune checkpoint inhibitors $\cdot$ Neuroendocrine tumors $\cdot$ Neuroendocrine neoplasia $\cdot$ Merkel Cell Carcinoma $\cdot$ Small Cell Lung Cancer

$\begin{array}{ll}\text { Abbreviations } \\ \text { CTLA-4 } & \text { Cytotoxic T-Lymphocyte Antigen } 4 \\ \text { PD-1 } & \text { Programmed death-1 } \\ \text { PD-L1 } & \text { Programmed death-1 ligand } \\ \text { ICIs } & \text { Immune checkpoint inhibitors } \\ \text { WHO } & \text { World Health Organization } \\ \text { FDA } & \text { Food and Drug Administration } \\ \text { ESMO } & \text { European Society for Medical Oncology }\end{array}$

Manuela Albertelli

manuela.albertelli@unige.it

Andrea Dotto

Endocrinology Unit, IRCCS AOU San Martino, Genoa, Italy

2 Endocrinology Unit, Department of Internal Medicine and Medical Specialties (DiMI), Centre of Excellence for Biomedical Research (CEBR), University of Genoa, Genoa, Italy

3 Medical Oncology Unit 1, IRCCS AOU San Martino, Genoa, Italy
IHC Immunohistochemical

NENs Neuroendocrine neoplasms

NECs Neuroendocrine carcinomas

NETs Neuroendocrine tumors

GEP Gastroeneteropancreatic

MCC Merkel cell carcinoma

SCLC Small cell lung cancer

LCNEC Large cell neuroendocrine carcinoma

GI-NENs Gastrointestinal neuroendocrine neoplasms

panNENs Pancreatic neuroendocrine neoplasms

TC Typical Carcinoid

AC Atypical Carcinoid

MCPyV Merkel cell polyomavirus

SRLs Somatostatin receptor ligands

PRRT Peptide receptor radionuclide therapy

TKI Tirosin Kinase Inhibitors

TMB Tumor mutational burden

Treg Regulatory T cells 


$\begin{array}{ll}\text { ADCC } & \begin{array}{l}\text { Antibody-dependent cell-mediated } \\ \text { cytotoxicity }\end{array} \\ \text { RCTs } & \text { Randomised Controlled Trials } \\ \text { CBR } & \text { Clinical benefit rate } \\ \text { DCR } & \text { Disease control rate } \\ \text { CR } & \text { Complete response } \\ \text { PR } & \text { Partial response } \\ \text { PD } & \text { Progressive disease } \\ \text { PFS } & \text { Progression-free survival } \\ \text { RFS } & \text { Recurrence-free survival } \\ \text { OS } & \text { Overall survival } \\ \text { ORR } & \text { Objective response rate } \\ \text { DOR } & \text { Durability of response } \\ \text { ES } & \text { Extensive-stage } \\ \text { AEs } & \text { Adverse events }\end{array}$

\section{Introduction}

While in the past the field of cancer therapy was dominate by surgery and radiotherapy associated with chemotherapy $[1$, 2], in the last decades research has provided new treatment strategies, such as target therapies. Target therapy, understood as use of drugs or other substances that targets specific molecules to arrest the growth and spread of cancer cells [3], has sharply revolutionized the outcomes of different types of cancer.

Currently, another intriguing weapon available in cancer therapy is represented by immunotherapy, based on the stimulation of the immune system against cancer cells through the introduction of cytokines and antibodies (passive immunotherapy) or the introduction of vaccines and immune cells themselves (active immunotherapy) [4]. Immune evasion mechanisms have a pivotal role for tumor cell proliferation and growth [5, 6]. The Cytotoxic T-Lymphocyte Antigen 4 (CTLA-4) [7] and the programmed death-1 (PD-1) and programmed death-1 ligand (PD-L1) [8] represent a key point regarding escape from immune surveillance by cancer cells, and this reason has led to the development of antibodies against these molecules. These antibodies, named 'immune checkpoint inhibitors' (ICIs), have opened a new era in oncology $[9,10]$. ICIs proved one of the most effective therapeutic approaches for several cancers. However, many patients are unresponsive to ICIs [11] and these immunotherapies can produce serious non-specific systemic inflammation and autoimmune side effects [12]. Therefore, the study of the tumor microenvironment has become fundamental [13] with the aim of identifying biomarkers to select the patients who can benefit most from these treatments.

Immunotherapy, so promising in many neoplasms, still does not have a precise role in the treatment of neuroendocrine neoplasms (NENs).
NENs constitute a heterogeneous group of rare tumors, which arise from enterochromaffin cells and can present throughout the body, however, in most cases, they are localized in the gastrointestinal tract, pancreas and thorax [14]. A rate amounting around $11-22 \%$ of all NENs are defined as unknown primary origin when their primary tissue of origin has not been identified with standard diagnostic work-up. Delineating the primary site of origin has important implications for selecting the appropriate treatment and overall prognosis. The small bowel, followed by the lung and pancreas are the most prevalent primary sites of origin in case of NEN with unknown primary. [15]

NENs display a highly variable biological features, clinical course and prognosis, making prediction of survival difficult. The most recent WHO 2019 classification [16], based on the WHO 2010 classification and extending the WHO 2017 classification [17], has established the importance of classifying gastroeneteropancreatic (GEP) NENs considering the primary site localization, the morphological differentiation, and the grading. According with these affirmations, GEP-NENs are graded into three different categories taking into account the Ki67 proliferation index. The real novelty of the last WHO 2019 classification is the G3 category, characterized by a Ki67 $>20 \%$ and a well differentiation morphology, extended to all GEP-NENs. Furthermore, it worth considering neuroendocrine carcinomas (NECs) represented by a Ki67 proliferation index $>20 \%$ and poorly differentiated.

The cornerstone of treatment in well-differentiated neuroendocrine tumors (NETs) is based on surgery, local ablative treatments, antisecretory and antiproliferative drugs, such as somatostatin receptor ligands (SRLs), peptide receptor radionuclide therapy (PRRT), and target therapies, while high-grade NECs benefit mostly of platinum-based cytotoxic chemotherapy [18]. However, new therapeutic strategies are being studied for the treatment of NENs, such as immunotherapy. In particular promising results have been especially observed in the treatment of two of most aggressive NENs with ICIs [19]: Merkel cell carcinoma (MCC), a rare very aggressive NET of the skin, and small cell lung cancer (SCLC). Few other types of neuroendocrine neoplasms are beginning to show interesting results [20].

In this article, we provide an overview on the current knowledge about immunotherapy applied to NENs, evaluating future perspectives in this setting.

\section{Methods}

We performed a literature search by MEDLINE (PubMed database) and we also considered the trials registered on clinicaltrials.gov to identify potentially relevant articles on immunotherapy with ICIs and NENs of any grade and primary site. The search was last updated 31 January 2021. 
The search strategy included the following terms "immunotherapy" OR "immune checkpoint inhibitor" OR "immune checkpoint blockade" OR "spartalizumab" OR "pembrolizumab" OR "toripalimab" OR "nivolumab" OR "ipilimumab" OR "atezolizumab" AND:

- GEP-NENs section: "gastroeneteropancreatic neuroendrocine neoplasms" OR "GEP-NENs" OR "gastroenteropancreatic neuroendocrine tumors"

- thoracic NENs section: "typical carcinoid" OR "atypical carcinoid" OR "large cell neuroendocrine carcinoma (LCNEC)" OR "SCLC"

- MCC section: "Merkel Cell Carcinoma" OR "MCC"

Only articles published in English were considered. Additional studies were identified by reviewing the references of all selected articles. The methods of potentially eligible studies were assessed independently by three reviewers (MA, $\mathrm{AD}, \mathrm{FN})$.

\section{Gastroeneteropancreatic neuroendocrine neoplasms (GEP-NENs)}

The incidence of NENs has risen in the last few decades. Especially, as for prevalence, GEP-NETs are the second neoplasm of the gastro-intestinal tract [21]. GEP-NENs arise in secretory cells of the gastrointestinal tract (GINENs) and pancreas (panNENs) [22, 23]. As for treatment, in well-differentiated GEP-NETs the first choice is local surgical treatment, when applicable. In case of metastatic NETs or surgical approach unenforceable, antiproliferative and antisecretory therapy with SRLs are recommended [18, 24]. Moreover, other strategies such as PRRT has been demonstrated effective in prolonging progression-free survival (PFS) especially in patients with GI-NETs [25]. Everolimus, sunitinib, and chemotherapy are further treatments investigated and approved for GEP-NENs. As regards GEP-NECs, cytotoxic chemotherapy platinum-based with etoposide is the most common available approach [26, 27]. It's worth considering the poor prognosis of these carcinomas with a median of about 7.5 months [28]. Furthermore, the therapeutic landscape for NENs has been evolving constantly and several clinical trials are ongoing to characterize immunotherapy as an emerging therapeutic strategy. In particular, ICIs, using anti-PD-1, anti-PD-L1 and anti-CTLA-4 agents, has been investigated in GEP-NENs in the last few years. Several studies have evaluated the expression of these checkpoints molecules in NETs of different grades and in NECs $[29,30]$. The potential a given NEN to respond to ICI is still largely unknown. Immunohistochemical (IHC) evaluation of PD-L1 expression in the tumor microenvironment and its role in predicting response to ICIs is a very burning topic. Moreover, a high tumor mutational burden (TMB) has been found associated to an increased benefit of ICIs [31-33] and was advocated as a possible useful biomarker to select potential responders to these drugs.

The importance of the immune microenvironment in patients with NETs has been established in the last years [34]. Immune cell infiltration is only documented in GEPNETs, and overall, it appears to be higher in panNETs rather than in midgut NETs, perhaps as a result of the higher TMB of panNETs [35, 36]. Morever, in another series of NENs, potentially immunosuppressive regulatory $\mathrm{T}$ cells (Treg) were present in $55 \%$ of intermediate/high-grade tumours, whereas only $16 \%$ of low-grade metastatic NENs showed intratumoral Treg $(\mathrm{P}=0.02)$. [37]. Nevertheless, more knowledge about the composite immune landscape of these heterogeneous tumors needs to be gained in order to clarify the prognostic implication that have these NENs features. [38].

Furthermore, even prognostic evidences linked with the expression of immune checkpoint molecules PD-L1 and PD-1 are still unclear and debated across different studies [39-42]. There are some data in the literature showing that PD-L1 expression was significantly correlated to a higher WHO grade of NENs [39, 42] and also with a poor PFS and overall survival (OS). On the contrary, others authors have found that PD-L1 expression did not correlate with grade or prognosis [40, 43]. In fact, recently, the expression of PD-L1 was investigated in a large cohort of patients with G3 GEP-NEN and only $10 \%$ of these tumors expressed PD-L1 and those lesions, with positive PD-L1 immunoreactivity in tumoral cells, were all poorly differentiated cases [43]. In this study no correlation was found between PD-L1 immunoreactivity and clinical parameters evaluated, such as age, sex, primary site, PFS and OS. Anyway, the design of the study did not include treatment intervention with ICIs, therefore the clinical outcome and the response to therapy could not be correlated with the expression of PD-L1 in this series [43].

As reported in different studies, TMB is typically low in well-differentiated lesions (NET G1 or G2) and higher in poorly differentiated carcinomas (G3), [34, 44].

Globally, all these studies have aroused new perspectives to initiate clinical trials aimed to identify the efficacy of ICIs in NENs.

\subsection{Pembrolizumab}

The non-randomized, phase $1 \mathrm{~b}$ trial KEYNOTE-028 investigated the safety and efficacy of the PD-1 inhibitor pembrolizumab, as monotherapy, in a large cohort of patients with advanced solid tumors with positive PD-L1 expression [45] As for NENs, 16 patients with pancreatic NEC were considered, one patient had objective response and 14 of them 
experienced stable disease. Interestingly, encouraging data emerged from the six-months follow-up demonstrating a PFS rate of $40 \%$. Furthermore, at the 12 -months evaluation, the PFS rate was of $27 \%$ and OS rate of $87 \%$. The following phase 2 KEYNOTE-158 trial, considering GEP-NETs, partial response was present in 3 patients with panNET and in 1 subject with rectal NET after a median follow-up of 24 months [46].

\subsection{Spartalizumab}

Spartalizumab is a humanized anti-PD1 monoclonal antibody, evaluated in a phase II, multicenter, single-arm study enrolling patients with well-differentiated metastatic G1/G2 NET (32 GI-NET; 33 panNET) and GEP-NEC. In the 21 patients with GEP-NECs a higher expression of PD-L1 in immune cells was observed respect than those with GEPNETs. As for the NETs cohort, the objective response rate (ORR) was 3,1\% in GI-NET and 3\% in panNET. These data did not accomplish the success label fixed as ORR $\geq 10 \%$ [47]. In the GEP-NEC group, ORR was $4.8 \%$ (95\% CI: 0.1, 23.8 ) and the 12 -month overall survival was $19.1 \%$. Interestingly, the ORR was higher in patients with higher PD-L1 expression or more CD8 + cells infiltration at baseline evaluation. Furthermore, spartalizumab determined limited adverse effects (observed in less than $50 \%$ of patients). In the GEP-NEC cohort less than $20 \%$ of subjects presented increased liver enzymes.

\subsection{Toripalimab}

Toripalimab is a humanized IgG4 antibody with human PD-1 receptor as target. This molecule was approved few years ago as second-line treatment in metastatic melanoma. A phase $1 \mathrm{~b}$ trial investigated its efficacy in patients with NENs recurring or metastatic after first-line therapy. Toripalimab schedule therapy was $3 \mathrm{mg} / \mathrm{kg}$ once every two weeks. [48]. In the cohort of 40 subjects, ORR was $20 \%$ and the median durability of response (DOR) was 15.2 months. Interestingly, in tumors characterized by PD-L1 expression $\geq 10 \%$ ORR was $50 \%$ while in those with PD-L1 $<10 \%$ ORR was $10.7 \%(\mathrm{p}=0.019)$.

\subsection{Ipilimumab and nivolumab}

The prospective, open-label, multicenter phase II clinical trial DART (Dual Anti-CTLA-4 and Anti-PD-1 Blockade in Rare Tumors, the trial is still ongoing, NCT02834013) investigated the efficacy and safety of the combined immunotherapy: ipilimumab plus nivolumab across multiple rare tumor cohorts. [49]. Ipilimumab combined with nivolumab were tested in a cohort of 32 patients with any grade of non panNETs. As for GI-NETs, 15 patients were enrolled and 18 out 32 patients had high-grade tumors. Results demonstrated a 44\% ORR in patients with NEC versus $0 \%$ in low and intermediate grade neoplasms. This combined therapy was investigated in another phase 2 trial, involving 29 patients with advanced NETs: overall, the ORR was $24 \%$ and as for panNETs $43 \%$ of patients experienced an objective response [50].

\section{Future perspective in GEP-NENs}

Evidence so far available for ICIs in GEP-NETs is definitively not as robust as for other tumors such as SCLC or MCC. We, therefore, had to take a further look to what the near future might reserve for ICIs in GEP-NET and NEC through an in-depth research on the clinicaltrials.gov database, considering only phase II or III studies, evaluating every Food and Drug Administration (FDA) approved ICIs molecule and not approved ones as well. Our research yielded 42 results, of which 19 were deemed relevant for our purpose; the results are summarized in Table 1; the considered drugs are used either in monotherapy, or in association with another ICIs, with Tirosin Kinase Inhibitors (TKI)/other target therapy, or with SRLs, or PRRT, or chemotherapy.

A total of nine different ICIs drugs are used throughout the 19 Randomised Controlled Trials (RCTs). Such numbers highlight the growing attention gathering around NENs and ICIs, in response to the need of stronger evidences supporting such therapy.

Some of the reported trials have, however, already produced initial results, most of them in the form of abstracts/ interim analyses: we retrieved seven initial reports from seven different RCTs, the majority of which showing promising results.

Four analyses report a clinical benefit rate (CBR) $>30 \%$ [51-54]. The first [51] is derived from NCT03043664, which investigates pembrolizumab plus SRLs in 22 patients with low/intermediate grade GEP-NETs. With this schedule around $40 \%$ of patients achieved stable disease (SD). Relevant antitumor activity was also described for avelumab, tested in 29 patients [52] deriving from NCT03352934, with either NEC or NET (mostly GEP), that obtained a disease control rate (DCR) of $32 \%$. Nivolumab combined with temozolomide showed promising results in an interim analysis [53] from NCT03728361: out of 12 patients (7 GEP-NETs and 5 pulmonary carcinoids) $25 \%$ showed partial response (PR), 67\% SD and $8 \%$ progressive disease (PD), although the median follow-up was short (approximately 7 months) and the cohort very limited. Lastly, according to an interim analysis [54] from NCT03365791, spartalizumab, administered with LAG525 (an antibody to LAG-3), achieved an astounding $86 \%$ of clinical benefit rate at 24 weeks in 


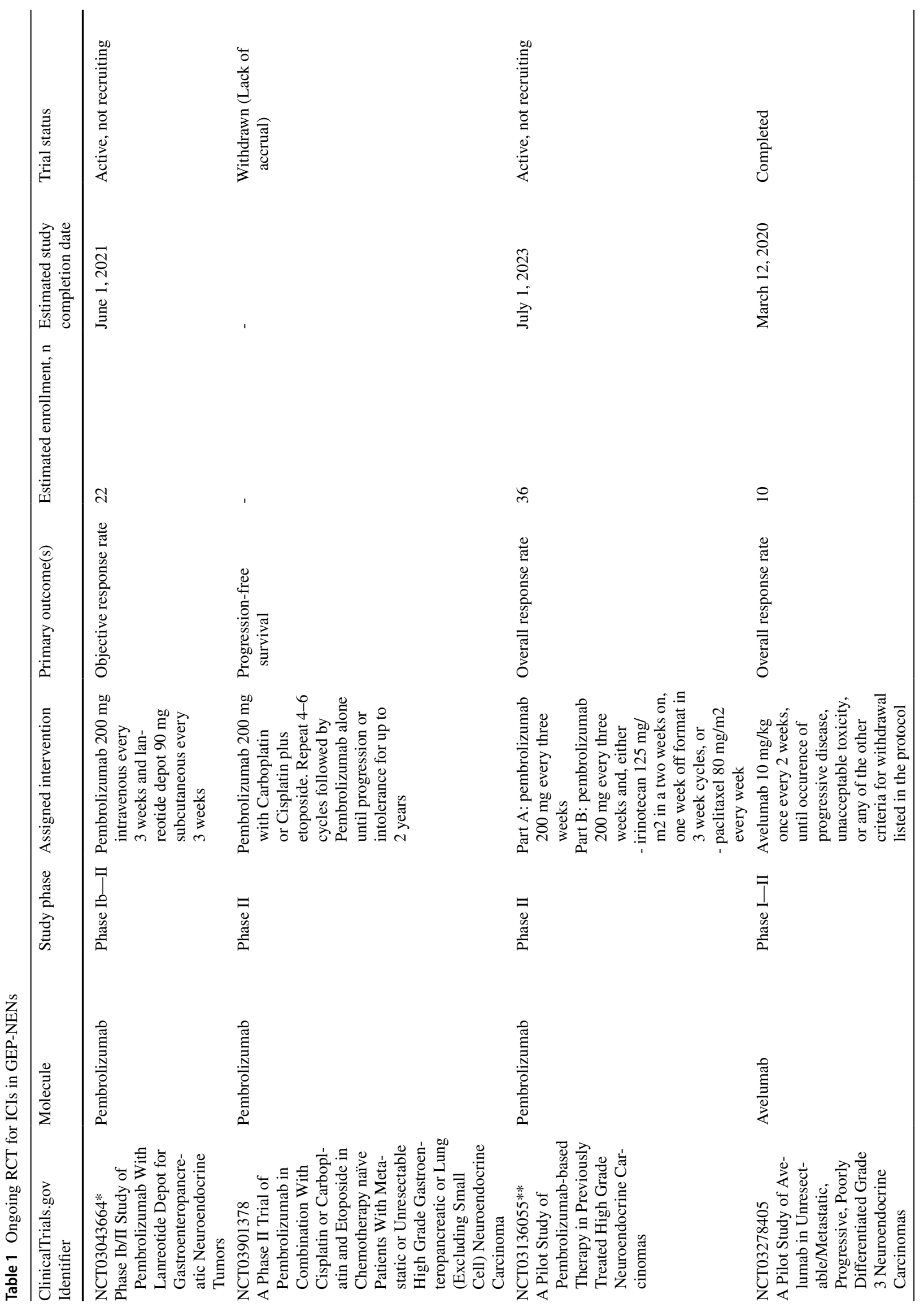




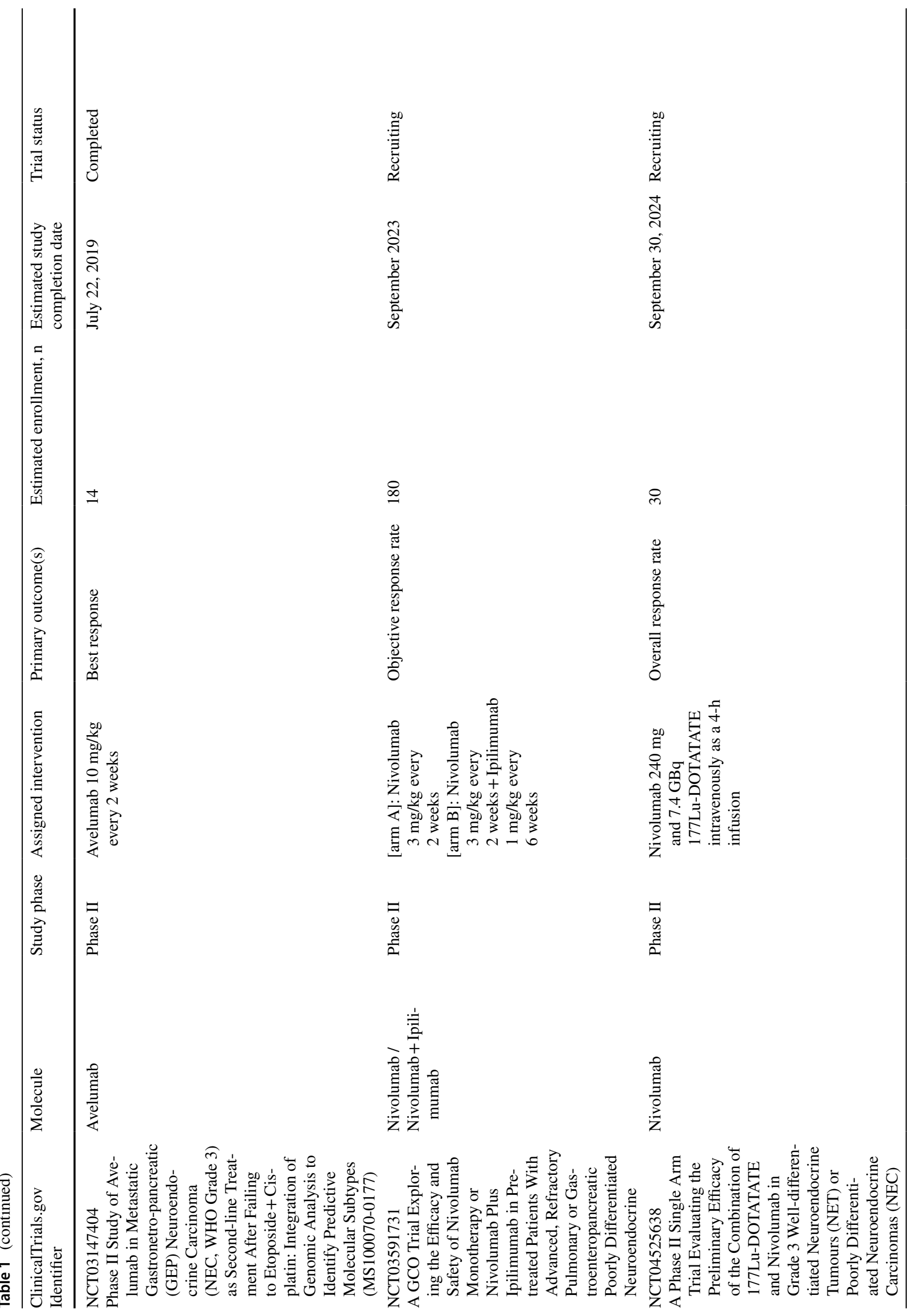




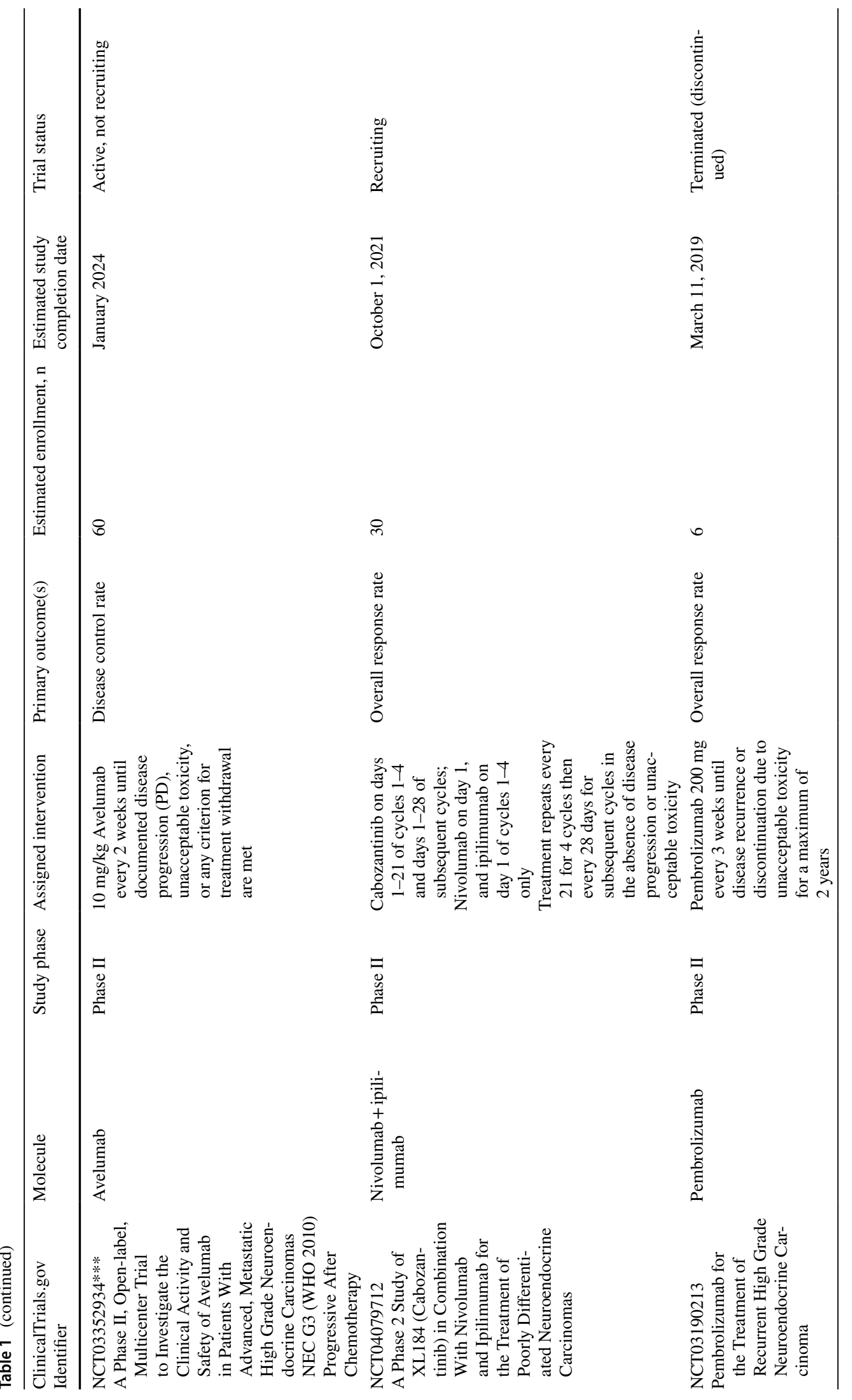




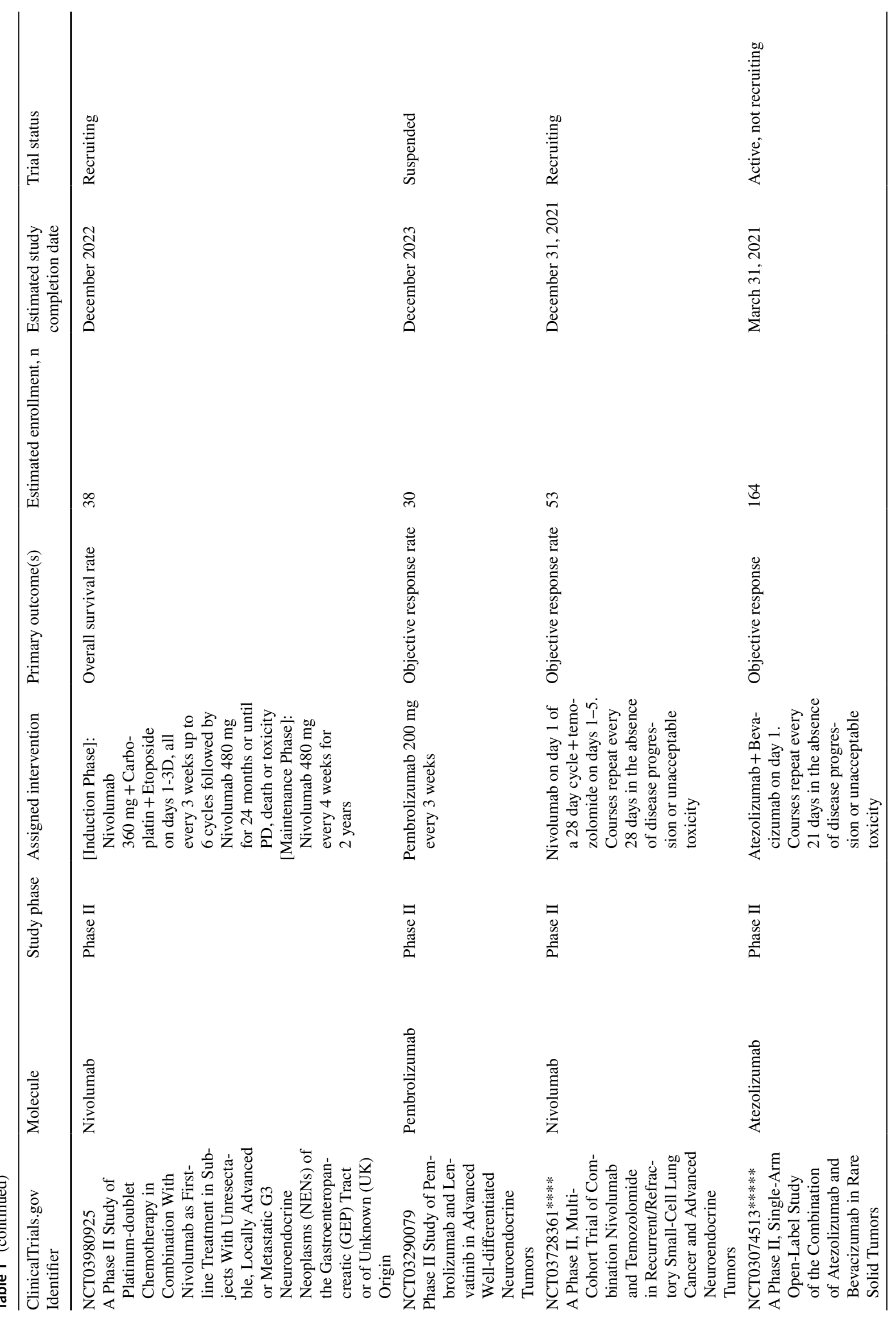




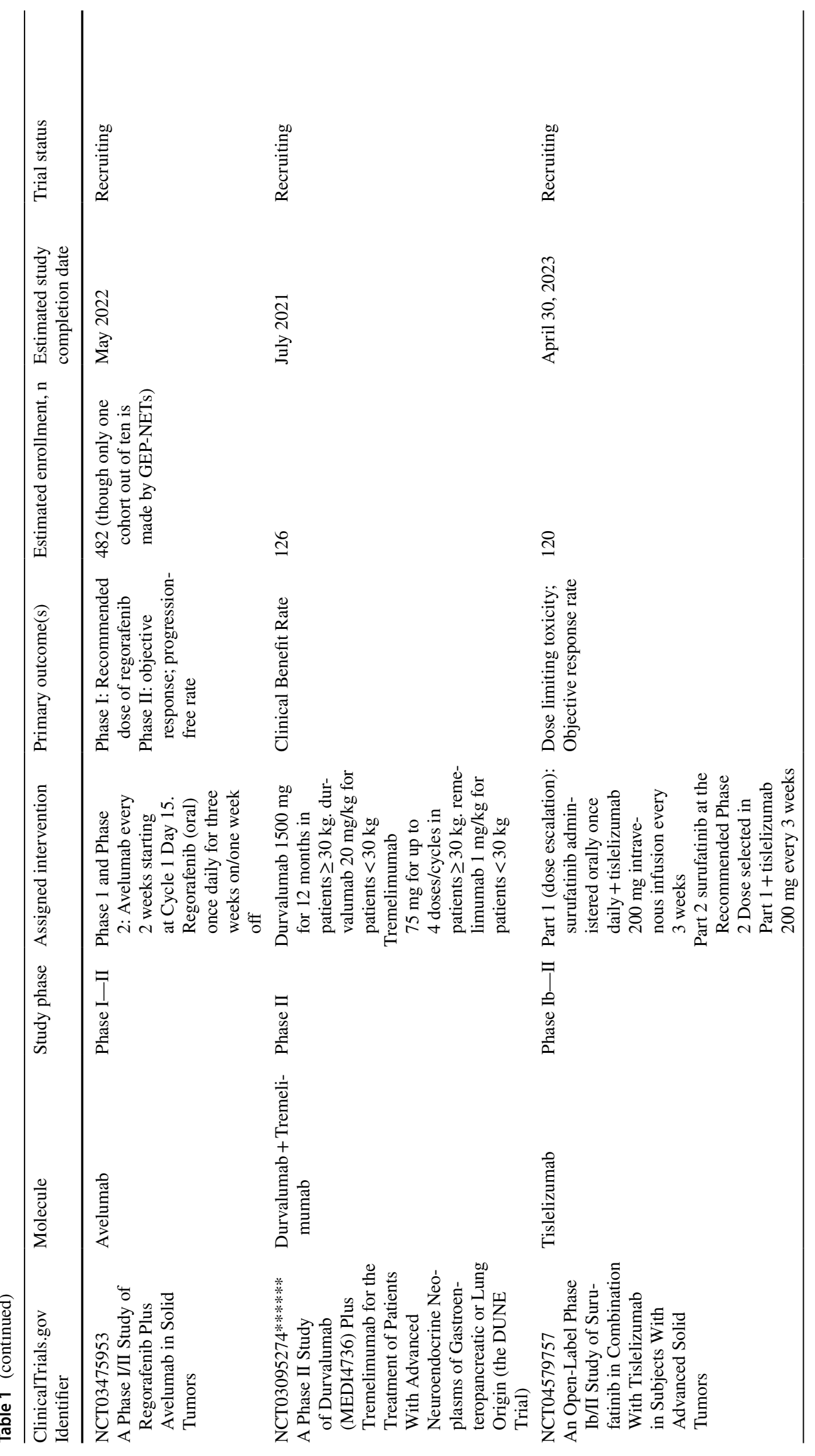




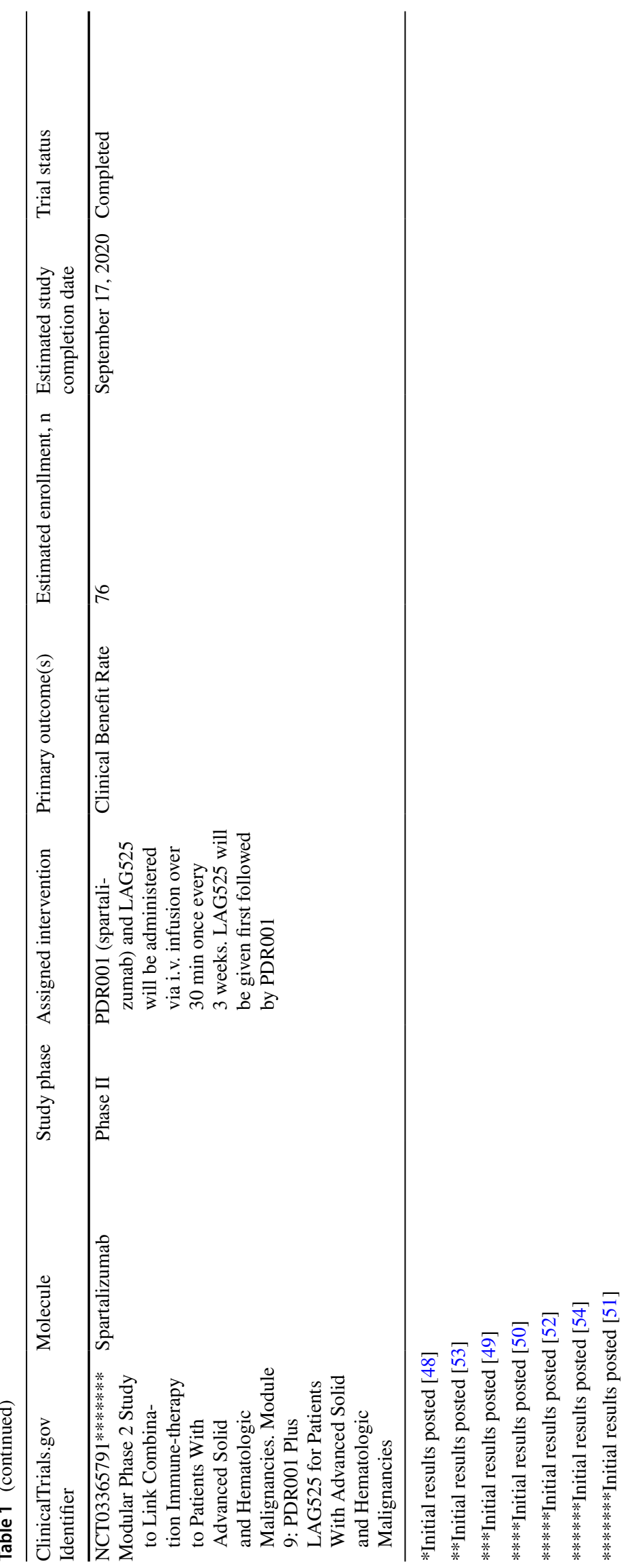


a GEP-NET cohort. With the exception of nivolumab plus temozolomide, the majority of the above mentioned analyses reported a good to an excellent tolerability as well.

More modest results are reported in another interim analysis [55] of NCT03074513, which aimed at defining the activity of atezolizumab plus bevacizumab in panNET and extra panNETs, achieving a confirmed objective response rate of $20 \%$ and $15 \%$ (95\% CI 3-38\%), respectively. This last association was also well tolerated. Worse results were, instead, reported in 14 patients with progressive NECs treated with pembrolizumab alone (partial results from NCT03136055). Pembrolizumab was deemed ineffective in this small cohort [56]. Similarly, durvalumab in combination with tremelimumab was almost ineffective the abstract [57] derived from NCT03095274: a global cohort of 123 patients was divided in subgroups considering the primary origin and grade (GI NETs, panNETs, GEP NECs and lung carcinoids, 123 patients in total) where a CBR $>30 \%$ at median followup of 10.8 months was observed only in the panNET group. Nor safety or tolerability concerns were registered.

As already mentioned, the results achieved in these RCTs should be considered partial because derived from studies including often a too low number of patients. However, considering the overall promising preliminary results observed, final results are eagerly waited for.

\section{Lung neuroendocrine neoplasms}

Lung NENs are a group of rare and heterogenous pulmonary tumors classified into four different histological groups following the 2015 WHO lung NEN classification [58]. Welldifferentiated lung NENs are typical (TC) and atypical (AC) carcinoids often characterized by indolent clinical behavior. On the other hand, pulmonary high-grade NENs are SCLC and large-cell neuroendocrine carcinoma (LCNEC). Briefly, TC is the most frequent lesion among the four histological groups and have a metastatic potential in up to $15 \%$ of patients with a median time to recurrence of 4 years. AC presents metastatic spread in up to one half of cases and has a median time to recurrence of 1.8 years. As for advanced lung NET, median survival from diagnosis is about $6-7$ years [59]. Concerning treatment, it worth considering a different approach based on the histological group and patients tailored. Since now, few trials have been designed for lung NETs specifically, therefore the therapeutic management largely derives from studies in patients with GEP-NENs [60]. In this view, the emerging immunotherapy strategies might represent a new base to change the therapeutic scenario of this group of NENs. Particularly, immunotherapy has become a paradigm shift in the treatment of SCLC. The presence of lymphocyte infiltration in lungs NENs have not been fully investigated so far. Researchers have obtained discrepant results as for the association between the host immune response to lymphocyte infiltration and the prognosis of lung NENs, especially in terms of OS and PFS [29].

\subsection{Lung carcinoid tumors: typical and a typical (TC and $A C$ )}

TC and intermediate-grade AC are heterogenous NENs whose treatment mostly derived from studies designed for GEP-NENs and SCLC. Nowadays, surgery is considered the first-choice treatment in local disease, whereas there is a lack of internationally approved therapies in case of metastatic carcinoids. Recently, immunotherapy has become a promising therapeutic option based on the emerging results regarding PD-1 and PD-L1 expression in lung NETs. It is worth quoting a recent paper enrolling 131 patients with TC and 37 with AC, in which immunohistochemistry was performed to detect the expression of PD-L1, PD-1, and CD8. As a result, PD-1 expression was present in 7\% of TC samples and no AC expressed PD-L1. Overall, the study pinpointed that carcinoids showed a low expression of both PD-1 and PD-L1. However, PD-L1 presence was strickling associated with mediastinal lymph-node metastasis at baseline. In this view, immunotherapy might represent a possible therapeutic option in the management of these neoplasms, undoubtedly to be investigated with further studies [61]. In this scenario, a recent case report described a patient with metastatic AC and persistent mediastinal lymphadenopathy refractory to first line treatment with platinum-based chemotherapy and etoposide. According with the results from the CheckMate-032 trial in SCLC, the combined therapy with nivolumab and ipilimumab was undertaken with a success tumor response [62].

Furthermore, the nonrandomized phase $1 \mathrm{~b}$ KEYNOTE-028 trial, evaluating a cohort of 25 patients with PD-L1-positive TC or AC treated with pembrolizumab, showed ORR of $12 \%$. However, a complete response (CR) was not observed in any patients of this cohort, whereas 15 subjects had stable disease, and 7 presented a progressive disease. At the 12 months follow-up, PFS rate was $27 \%$ and ORR 65\%. Median OS was 21.1 months (9.1 to 22.4 months) [45].

Moreover, spartalizumab was evaluated in a phase II trial enrolling 116 patients with advanced NETs, of different primary origin, and GEP-NEC, in progression after previous treatments. As regards well-differentiated NETs, the median follow-up was 7.6 months, with an overall ORR of $7.4 \%$. Interestingly, thoracic NETs had a higher ORR (20\%). In the thoracic carcinoid cohort, stable disease was reached in $53.3 \%$. PD-L1 expression was higher in GEP-NECs compared to the thoracic carcinoids (43\% vs. 19\%) [47].

The ongoing trial dual anti-CTLA-4 and anti-PD-1 blockade in rare tumors (DART SWOG 1609), has gathered 32 
patients with non-pancreatic NENs, and 6 of them with lung as primary origin. These patients were treated with nivolumab combined with ipilimumab and the overall ORR observed was $25 \%$. Whereas, considering low-intermediate grade neoplasms, ORR was 0\% [49].

\subsection{Large cell neuroendocrine carcinoma (LCNEC)}

LCNEC is a rare carcinoma and accounts for less than $1 \%$ of all lung tumors. It has a poor prognosis, with 5-year overall survival rates of about $13-57 \%$. Treatment strategies include surgical approach, radiotherapy, and cytotoxic chemotherapy. Evidences of immunotherapy in these neoplasms are scares and derive especially from cases reports and retrospective analysis. Briefly, an interesting case report pinpointed the remarkable tumor response to nivolumab in a second-line setting, in a young woman with a locally advanced LCNEC (cT4 cN2 cM0 at baseline vs. ycT2a ycN0 ycM0 after 14 cycles) [63]. Similarly to this case, in the literature are present other papers highlighting the response to PD-1 inhibitors, especially nivolumab, as second- or thirdline treatment in patients with LCNEC. The larger analysis available, included 10 patients with advanced LCNEC ( 8 patients had a stage IV disease) treated with nivolumab or pembrolizumab as second-line or further (first line therapy was platinum-based chemotherapy). Overall, $60 \%$ of patients had a partial response and in one case a stable disease was reached. Median PFS was 57 weeks and $80 \%$ of patients stopped immunotherapy because of disease progression and only one for a pulmonary interstitial pneumonia [64, 65]. There are not significant evidences regarding immunotherapy with PD-1 inhibitors and/or CTLA4 inhibitors as first-line approach in LCNEC.

\subsection{Small cell lung cancer (SCLC)}

SCLC is a high-grade NEN characterized by a biological aggressiveness like that of LCNEC, early spread to distant metastases or metastatic disease at the time of diagnosis, and a negative prognosis with a poor OS. As regards localized disease, median survival has been reported to be $15-20$ months, and only $20 \%-25 \%$ of patients survived after 5 years from diagnosis. According with ESMO clinical practice guideline, chemotherapy with platinum and etoposide is the first-line approach, while as second-line treatment topotecan has been approved [66]. Among NENs, SCLC is one of the most studied tumours and data on its biology have led to possible new therapeutic options. Since now, a high TMB has been identified, opening the scenario of immunotherapy due to its predictive role as possible biomarkers of response. Moreover, even if SCLCs scarcely express immune-checkpoint molecules, ICIs with a promising clinical activity are currently under investigation [67].
In this view, immunotherapy has been evaluated in many trials exploring its role in different settings, such as first-line therapy or second- or further line, as well as in monotherapy or in combined schedules, with the scope of leading to an innovative management of this high-grade neoplasms.

\subsubsection{Atezolizumab}

The FDA approved few years ago atezolizumab in combination with carboplatin and etoposide as first-line treatment of adult patients with extensive-stage (ES) SCLC. Approval was based on the IMpower133 randomized, multicentre, double-blind, placebo-controlled trial enrolling more than 400 patients with ES SCLC who received no prior chemotherapy for extensive stage disease. Atezolizumab plus carboplatin and etoposide, for a maximum of 4 cycles, followed by atezolizumab as maintenance until disease progression or unacceptable toxicity, were compared to placebo and carboplatin and etoposide for a maximum of 4 cycles, followed by placebo until disease progression or unacceptable toxicity. Efficacy was measured by an outcome showing a median OS of 12.3 months $(10.8,15.9)$ for patients receiving atezolizumab with chemotherapy and 10.3 months (9.3, 11.3) for those receiving placebo with chemotherapy (hazard ratio 0.70; 95\% CI: 0.54, 0.91; $p=0.0069)$. Median PFS was 5.2 months $(4.4,5.6)$ compared with 4.3 months $(4.2,4.5)$ in the atezolizumab and placebo arms, respectively (HR 0.77; $0.62,0.96 ; p=0.0170)$. The most common adverse reactions, reported in $\geq 20 \%$ of patients who received atezolizumab, were asthenia, nausea, alopecia, constipation and decreased appetite [68].

Furthermore, atezolizumab versus conventional chemotherapy has been investigated as second-line therapy in 73 patients prior treated with platinum-based chemotherapy. Patients were not selected considering PD-L1 expression. The primary endpoint was objective response rate at 6 weeks, experienced by 1 of 43 eligible atezolizumab patients $(2.3 \%, 95 \%$ confidence interval $[\mathrm{CI}]: 0.0-6.8)$. Median PFS was 1.4 months (95\% CI: 1.2-1.5) with atezolizumab and 4.3 months (95\% CI: 1.5-5.9) with chemotherapy. OS did not significantly differ between groups. Median OS was 9.5 months versus 8.7 months for the atezolizumab and the chemotherapy group, respectively (adjusted hazard ratio atezolizumab: $0.84,95 \% \mathrm{CI}: 0.45-1.58 ; \mathrm{p}=0.60$ ) [69].

\subsubsection{Durvalumab}

Durvalumab, an IgG1 kappa anti-PD-L1 monoclonal human antibody, in combination with etoposide and either carboplatin or cisplatin has been recently approved by FDA as first-line treatment of patients with ES-SCLC. Approval was based on data from CASPIAN randomized, multicentre, active-controlled, open-label trial. Patients with untreated 
ES-SCLC were randomized to durvalumab plus chemotherapy vs. chemotherapy alone. The compelling result was concerning OS with a median of 13.0 months $(95 \% \mathrm{CI}$ : $11.5,14.8$ ) in the durvalumab plus chemotherapy arm compared with 10.3 months $(95 \%$ CI: 9.3, 11.2) in the chemotherapy alone arm (hazard ratio 0.73 ; 95\% CI: $0.59,0.91$; $\mathrm{p}=0.0047)$. PFS ( $96 \%$ of total planned events) showed a HR of 0.78 (95\% CI: $0.65,0.94)$, with median PFS of 5.1 months (95\% CI: 4.7, 6.2) in the durvalumab plus chemotherapy arm and 5.4 months $(95 \% \mathrm{CI}: 4.8,6.2)$ in the chemotherapy alone arm. ORR was $68 \%$ (95\% CI: 62\%, 73\%) in the durvalumab plus chemotherapy arm and 58\% (95\% CI: 52\%, 63\%) in the chemotherapy alone arm. Adverse reactions experienced by more than $20 \%$ of patients with ES-SCLC were nausea, asthenia and alopecia [70].

Moreover, the CASPIAN phase III trial also evaluated the combined therapy with durvalumab plus tremelimumab, an anti-CTLA-4 human monoclonal IgG2 antibody, plus platinum-etoposide followed by durvalumab as maintenance, until disease progression or unacceptable toxicity. Results from this combined therapy are not available yet [71].

\subsubsection{Ipilimumab}

The efficacy and safety of ipilimumab, an anti-CTLA4 inhibitor, was investigated in a randomized, double blind phase III trial without any evidence of prolonged OS with the combination of ipilimumab with chemotherapy. Indeed, 1132 patients with a diagnosis of ES-SCLC received as first-line ipilimumab or placebo plus etoposide and platinum. Median OS was 11.0 months for chemotherapy plus ipilimumab versus 10.9 months for chemotherapy plus placebo (hazard ratio, $0.94 ; 95 \% \mathrm{CI}, 0.81$ to $1.09 ; \mathrm{P}=0.3775$ ). Median PFS was 4.6 months in the chemotherapy plus ipilimumab arm versus 4.4 months in the chemotherapy plus placebo arm (hazard ratio, 0.85; 95\% CI, 0.75 to 0.97). Observed adverse effects were comparable in the 2 arms but it is worth highlighting that diarrhea, rash, and colitis, were more represented in the ipilimumab arm [72].

\subsubsection{Nivolumab monotherapy or combined therapy Nivolumab plus Ipilimumab}

As for first line-maintenance, the phase III trial CheckMate-451, enrolling 834 patients with not progressed ES-SCLC after first-line platinum-based chemotherapy, investigated nivolumab, an IgG4 anti-PD1 human monoclonal antibody, and the combined therapy with nivolumab plus ipilimumab. Primary endpoint was OS for nivolumab plus ipilimumab versus placebo and secondary endpoints included OS for nivolumab versus placebo. Initial results from a 2019 ESMO abstract did not show an improved survival with median OS of 10.4 vs. 9.2 vs. 9.6 months in the nivolumab, nivolumab combined with ipilimumab, and placebo arms, respectively. Considering the side effects observed, the higher rate of all grade of them was reported in the nivolumab plus ipilimumab arm (86\%) [73].

As regards II-line approach, the open-label, randomized phase III trial CheckMate-331 failed to show an improved OS comparing nivolumab versus chemotherapy (topotecan or amrubicin) in patients with relapsing SCLC after firstline platinum-based chemotherapy. Indeed, median OS was 7.5 months in the nivolumab group and of 8.4 months in the control one. The median PFS was 1.5 vs. 3.8 months (HR 1.41 (95\% CI: 1.18-1.69)) in the nivolumab and the topotecan arms, respectively. The evidence to pinpoint is the rate of adverse effect: all grade $55 \%$ in the nivolumab arm and $90 \%$ in the control arm [74]. Nivolumab alone and nivolumab plus ipilimumab in recurrent SCLC were investigated in the CheckMate-032 multicentre, open-label, phase $1 / 2$ trial. Enrolled patients had at least already completed first-line platinum-based chemotherapy, and most of them also other line of therapy. The schedule as second or further approach was nivolumab or nivolumab plus ipilimumab for four cycles, followed by nivolumab until disease progression or unacceptable toxicity. ORR was $10 \%$ in patients treated with nivolumab monotherapy, $23 \%$ in the arm receiving nivolumab $1 \mathrm{mg} / \mathrm{kg}$ plus ipilimumab $3 \mathrm{mg} / \mathrm{kg}$, and $19 \%$ in that receiving nivolumab $3 \mathrm{mg} / \mathrm{kg}$ plus ipilimumab $1 \mathrm{mg} /$ $\mathrm{kg}[75]$.

Considering monotherapy as a third-line treatment, FDA approved nivolumab for patients with metastatic SCLC with progression after platinum-based chemotherapy and at least one other line of therapy. Approval was based on data concerning the ORR in a subgroup of patients from CheckMate-032. Indeed, 109 patients with metastatic SCLC, regardless of tumour PD-L1 expression, were treated with nivolumab monotherapy in a third-line setting. The ORR was $12 \%$ (95\% CI: 6.5, 19.5). Responses were durable for 6 months or longer in $77 \%, 12$ months or longer in $62 \%$, and 18 months or longer in $39 \%$ of the 13 responding patients. PD-L1 tumour status seemed not to predict response in this cohort. As for safety, serious adverse reactions occurred in $45 \%$ of patients. The most frequent $(\geq 2 \%)$ serious events were pneumonia, dyspnoea, pneumonitis, pleural effusion, and dehydration [76].

\subsubsection{Pembrolizumab}

Pembrolizumab is an IgG4 anti-PD-1 monoclonal antibody. Its efficacy and safety in patients with SCLS were evaluated in a second-line approach. An interim analysis of the KEYNOTE-158 (a phase 2 basket study comprizing 11 different cancer types) investigated pembrolizumab in 107 patients with advanced SCLC treated with chemotherapy as firstline and evidence of disease progression or intolerance to 
the aforementioned standard therapy. Tumor expression of PD-L1 was also assessed. Median OS was 9.1 months (95\% CI, 5.7-14.6), 14.6 months in patients with PD-L1-positive tumors, and 7.7 months (95\% CI, 3.9-10.4) neoplasms without PD-L1 expression. As results, pembrolizumab has to be further investigated, especially in patients with advanced SCLC and tumor samples positive for PD-L1 expression [77].

FDA approved pembrolizumab for patients with metastatic SCLC and evidence of disease progression on or after platinum-based chemotherapy and at least one other prior line of therapy. Approval was based on data derived from a pooled analysis gathering 83 patients from the SCLC group of both KEYNOTE-158 and KEYNOTE-028 trials. Patients received pembrolizumab until documented disease progression, unacceptable toxicity, or a maximum of 24 months. ORR was $19 \%$ (95\% CI: 11,29$)$. The CR rate was $2 \%$ and responses were durable for 6 months or longer in $94 \%$, 12 months or longer in $63 \%$, and 18 months or longer in $56 \%$ of the 16 responding patients. The drug was discontinued for adverse reactions in $9 \%$ of patients and serious adverse events (AEs) occurred in 31\% [78].

Furthermore, pembrolizumab was investigated combined with paclitaxel in 26 patients with etoposide/platinumrefractory ES-SCLC showing a moderate activity with acceptable toxicity. ORR was $23.1 \%$, median PFS and OS were 5.0 months (95\% CI: 2.7-6.7) and 9.1 months (95\% CI: $6.5-15.0)$, respectively [41].

\section{Merkel cell carcinoma}

MCC is a rare skin malignant tumor first described in 1972 [79], which neuroendocrine origin has been hypothesized only in 2012 [80]. Although being a rare neoplasm, it is responsible for the higher number of skin cancer-related death per year after melanoma. About $80 \%$ of the cases is driven by the infection of Merkel cell polyomavirus (MCPyV) [81], therefore being the major risk factor, while other risk factors for the development of MCC include UV irradiation, and immunodeficiency conditions.

Since patients showing a particularly strong immune response have been found to have more favorable prognoses [82], the possibilities of immunotherapy for MCC appear to be greater than in other types of neoplasms, including other NENs.

Indeed, therapy with ICIs for MCC is certainly proceeding at a faster pace than for the majority of other NENs, and evidence from literature concerning MCC is more robust.

The position of immunotherapy in the treatment algorithm of metastatic/advanced MCC is beginning to gain more and more consideration, since chemotherapy, despite showing satisfying response rates, cannot guarantee a durable response [83]. Considering that some chemotherapy regimens can further worsen the impairment of the patient's immune system, and given the pivotal role of such factor when speaking of MCC, attention towards different approaches is steadily growing, and ICIs are drawing much of such attention.

As said, the risk of developing MCC is increased from five to 50-fold in persons with proven T-cell dysfunction, such as HIV-positive patients, solid organ transplant recipients, patients affected by hematological malignancies, and other immune system-impairing situations. Indeed, immunocompromised individuals represent approximately $10 \%$ of all the MCC patients [82, 84, 85]. Furthermore, immunosuppression represents a dramatic negative prognostic factor for mean survival, with patients showing any degree of immune system dysfunction showing an OS at three years approximately half of immunocompetent individuals (40\% vs 74\%) [86]. Conversely, better outcomes have been observed for patients showing particularly strong immune response features, such as highly $\mathrm{CD} 8^{+}$lymphocytes infiltrated tumors and MCCs showing no primary (MCC of unknown primary), which have higher tumor mutational burden and drive strong immune response $[87,88]$.

It comes of no surprise, though, due to its peculiar carcinogenesis, that MCC is indeed a highly immunogenic neoplasm.

As a matter of fact, it has been observed that both the infection by MCPyV and the DNA damage from UV radiation exposure (and the consequent high tumor mutational burden) lead to an increased response by the patient's immune system [89].

MCPyV-positive MCCs express virus-related oncoproteins that eventually will drive unregulated growth [90]. Such proteins cause the patient's immune system response in terms of intratumoral infiltration of CD8 + lymphocytes, the presence of MCPyV-specific T cells in the peripheral blood stream and IgG antibodies against said antigens [91, 92].

Similar pattern has been shown also in virus-negative MCCs, where the significant mutational burden caused by UV-radiation leads to production of neo-epitopes and proteins that are recognized by the patient immune system [93]. The presence of the aforementioned markers of immune system activation are all predictors of better prognosis [82].

Where it is true that a robust immune system activation is a favorable prognostic factor, it is also true that MCC can develop methods to escape the patient's immune system. For example, MCCs thwart NK and T-cell-mediated oncolysis is by downregulating Toll-like receptor $9 \mathrm{MICA} /$ MICB and MHC. A pivotal role in escaping lymphocytemediated eradication is also represented by PD-1 signaling: the tumor microenvironment, through the action of type II interferons, cause the overexpression of PD-L1 by the cancer cells, contributing to a decrease in the ability of inducing tumor cell death. Moreover, CD8 + T-cells infiltrating the 
tumor frequently overexpress PD-1 protein in response to persistent viral or tumor antigen exposure, as a marker of T-cell exhaustion [94].

PD-1 signaling appears to play a fundamental role in MCC; moreover, the highly immunogenic nature of this tumor provides a credible rationale for treatment with ICIs. To date, the only two ICI molecules FDA-approved for MCC are avelumab and pembrolizumab. [95, 96]; nevertheless, studies and trials concerning different ICIs are starting to populate the scientific panorama and initial evidence is available also for them eligible papers are summarized in Table 2.

\subsection{Avelumab}

This drug is rather unique amongst the ICIs since, beside its anti-PD-1/PD-L1 activity, it can mediate antibodydependent cell-mediated cytotoxicity (ADCC). Anyway, no proved lysis of activated immune cells from avelumab has been observed so far $[95,97]$. It was granted accelerated approval by FDA specifically for the treatment of metastatic MCC in adults and pediatric patients at least 12 years old, regardless of previous chemotherapy, subsequently to results produced in the JAVELIN Merkel 200 trial. In this study 88 patients with stage IV chemotherapy-refractory MCC were enrolled and treated with avelumab. ORR was $33.0 \%$ and responses were found to be durable, with $74 \%$ lasting more than 1 year. ORR was associated with a $94 \%$ risk reduction of death at 18 months. Beside such consistent results, avelumab was well tolerated, with only $5 \%$ of the treated patients developing grade $3 \mathrm{AEs}$ and no patients experiencing a grade 4 AEs, with an improved quality of life. [98, 99] As expected, patients with PD-L1 expressing neoplasms had better response [100].

Following these findings, initial results from an interim analysis of the ongoing phase III JAVELIN Merkel 200 trial part B where avelumab was used for MCC as first line therapy were released in 2018, showing an ORR of $62.1 \%$ with 14 of 18 responses (77.8\%) ongoing at the time of analysis. In the responding group, the estimated duration of response of at least 6 months was a remarkable $83 \%$, with no grade 4 or 5 adverse event registered [101].

Real life experience is so far limited, nevertheless evidences have already been produced by Walker et al. [102] in their study including almost 500 patients with by either MCC or progressive MCC. The authors confirmed the positive results seen in the JAVELIN trial. In fact, among 240 evaluable patients, ORR was $46.7 \%$ and DCR $71.2 \%$, with almost $23 \%$ of CR. Mean treatment duration was 7.9 months and, surprisingly, out of 16 immunocompromized patients, 11 (68.8\%) experienced DCR, and ORR was (37.5\%). These results were comparable to those recorded in the non-immunocompromized cohort, therefore opening a potential new window also for this category.

\subsection{Pembrolizumab}

Recently, in late 2018, pembrolizumab gained approval by FDA for treatment of recurrent locally advanced or metastatic MCC, based on the KEYNOTE-017 trial, a multicenter, nonrandomized, open-label trial that enrolled 50 patients with recurrent locally advanced or metastatic MCC who had not received prior systemic therapy for their advanced disease [103, 104].

In KEYNOTE-017 trial 50 patients with recurrent locally advanced or metastatic MCC and no prior systemic chemotherapy were enrolled. According to the protocol, patients received pembrolizumab as a first-line treatment for 2 years or until PD or withdrawal for toxicity. Of such cohort, $68 \%$ had PD-L1 positive neoplasms. Overall response rate was $56 \%$, with $24 \%$ experiencing CR and $32 \%$ PR, with ORRs of $59 \%$ in virus-positive and $53 \%$ in virus-negative tumors at a median follow-up time of 14.9 months.

While in the avelumab trial patients with tumor expressing PD-L1 displayed a better response, in the pembrolizumab study such a difference was not significant, however a positive trend in this direction was observed.

\subsection{Nivolumab}

The results of nivolumab from clinical trials in patients with MCC are only partial so far and are mostly derived from the CheckMate-358 study (NCT02488759, Non-Comparative, Open-Label, Multiple Cohort, Phase 1/2 Study of Nivolumab Monotherapy and Nivolumab Combination Therapy in Subjects With Virus-Positive and Virus-Negative Solid Tumors).

In 2017, an abstract containing data from 25 patients enrolled was released [105]. The response, evaluable in 22 out of 25 patients ( $71 \%$ treatment-naïve patients), showed an ORR of $68 \%$. At three months, PFS and OS rates were $82 \%$ and $92 \%$, respectively, with $3 / 4$ AEs occurring in $20 \%$ of patients (and in $12 \%$ of the patients led to therapy discontinuation).

More recently, in 2020, a paper, reporting the results of a neoadjuvant treatment in a cohort of MCC-patients (enrolled in the CheckMate 358), was published [106]. Out of the 36 patients who did undergo surgery, $47 \%$ achieved $\mathrm{CR}$ and $55 \%$ of the 33 radiographically evaluable patients who underwent surgery had at least tumor reduction $\geq 30 \%$. Recurrence-free survival (RFS) and OS were not reached at 20.3 months.

The CheckMate-358 study is still ongoing, with its estimated completion date set for August 2022. 


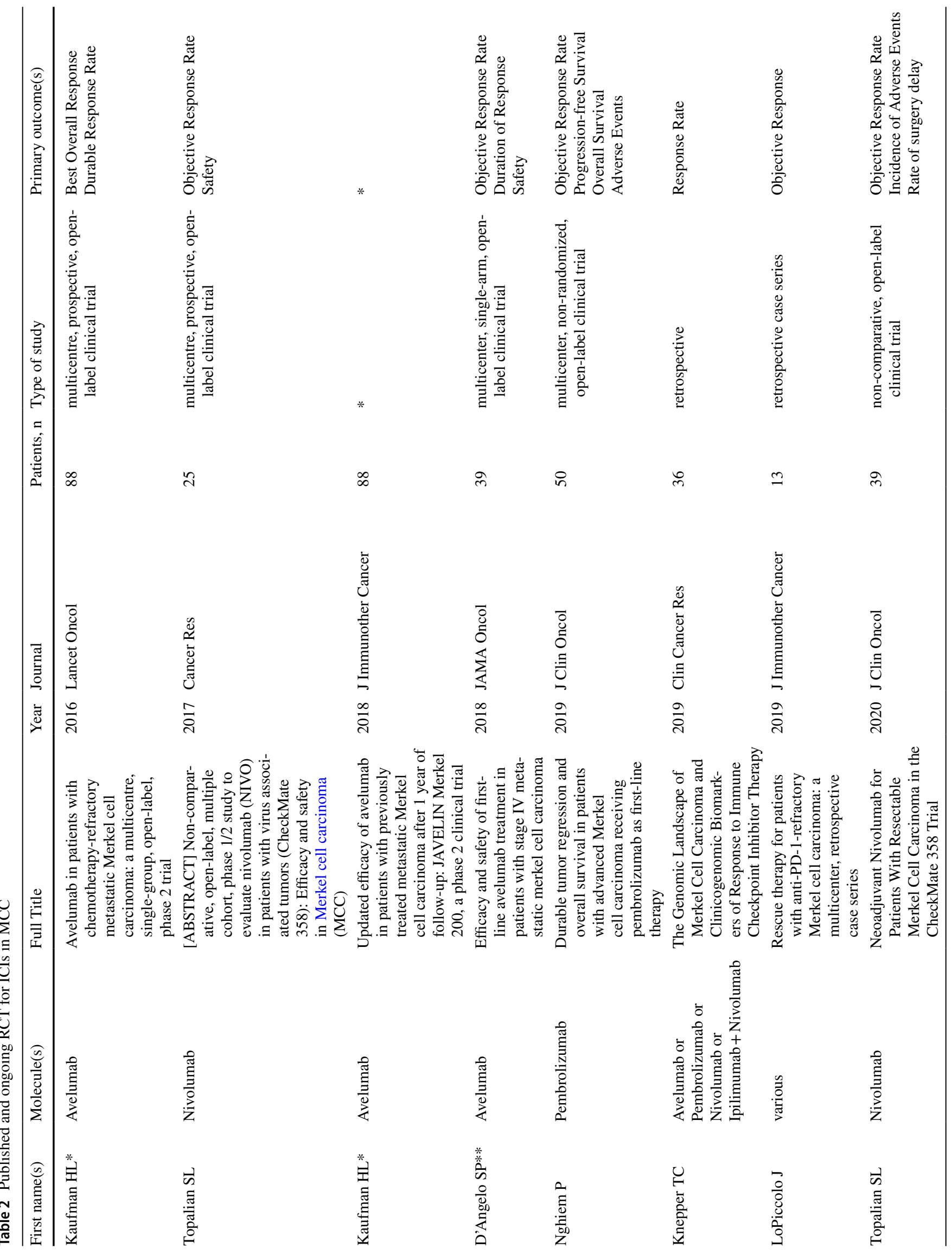




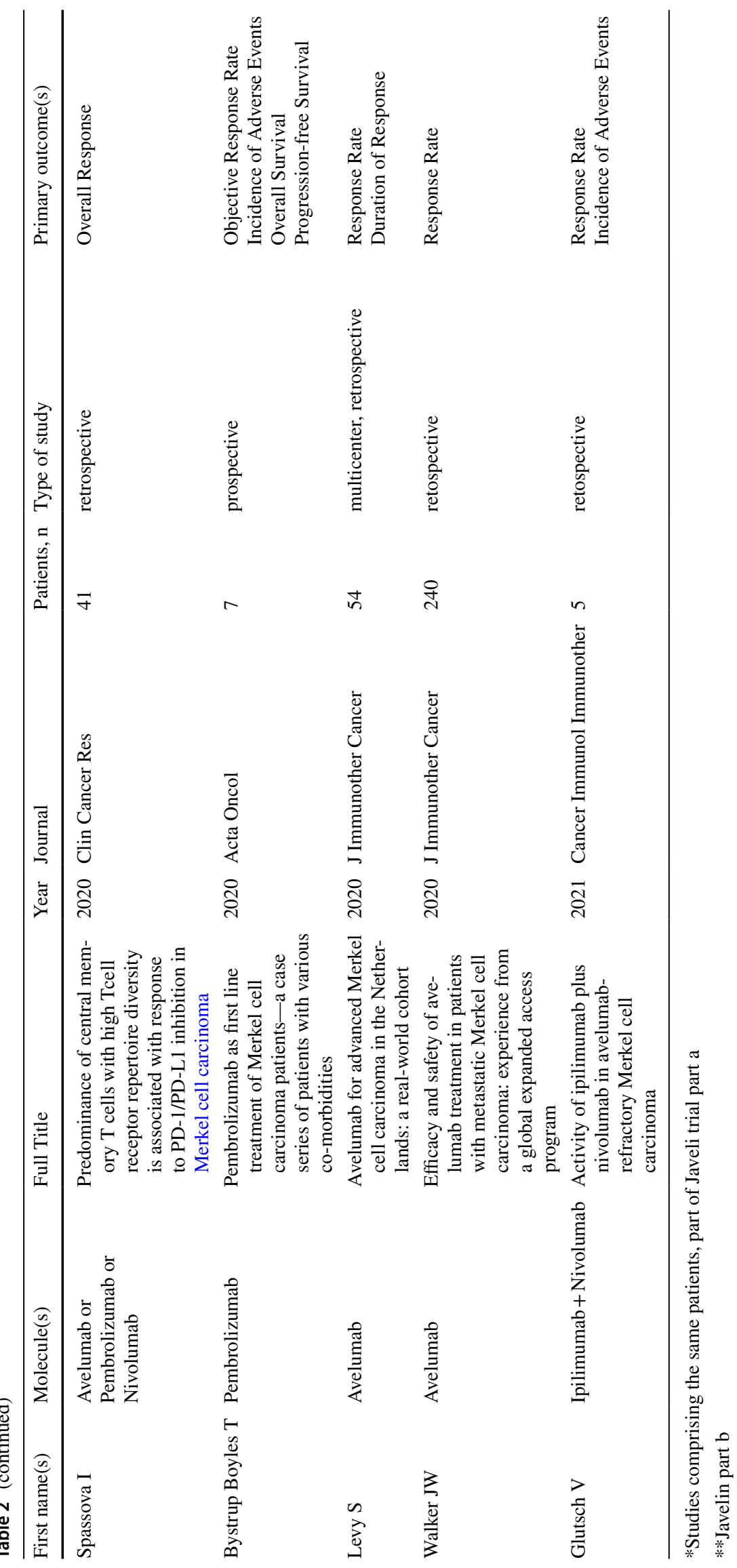




\subsection{Real-life Data}

Such dazzling results from clinical trials, the most interesting one being probably the sustained durability response (especially when compared to chemotherapy), together with consistent response rates and acceptable safety profiles, already gained ICIs approval as recommended firstline treatments for metastatic MCC in countries such as US and Germany [107].

While initial evidence is available from clinical trials, fewer real-life data are available on ICIs in MCC patients. Few authors have described experience with such drugs in MCC so far [108-111]. In the mentioned real-life studies, which comprise cohorts of patients treated with either avelumab, pembrolizumab or nivolumab plus ipilimumab, response rates ranged between $39-57 \%$, with the best one found in a cohort of only avelumab-treated patients [111]. Some authors also included immunocompromised patients in their studies. Knepper et al. [108] found significant relationship between the number of lines of therapy and response, with a $75 \%$ rate for first-line, $39 \%$ and $18 \%$ in second and third, respectively. Spassova et al. [109] in their retrospective study found that immunocompetent patients responded better than ones with hematological malignancies or under immunosuppressive drugs, whereas the type of drug was not found to be predictor of response. Levy et al. [111] showed that for avelumab, real-life data appear to be in line with results from clinical trials in terms of efficacy and toxicity. DOR in their cohort was 8.4 months, but with $43 \%$ of the patients having ongoing response at the end of the study. In the small retrospective series of patients treated with first-line pembrolizumab reported by Bystrup Boyles et al. [110], DOR was confirmed to be consistent, ranging from 16.3 to 27.1 months at cut off, but toxicity was found to be much higher compared to clinical trials data.

Data of efficacy of first-line ICI-based therapy appear to be promising, but still a consistent portion of patients either do not respond to the chosen ICI or develop resistance. Since chemotherapy does not provide a sustained response over time, the possibility, after failure of the first one, of a second-line ICI-therapy with a different target is definitively a possibility worth to be discussed. So far, few studies have investigated said option: LoPiccolo et al. [112] reported a case series of 13 patients treated with ICIs after experiencing PD under anti-PD-1 therapy. OR was observed in $31 \%$ of patients, with one patient refractory to anti-PD-1 therapy experiencing tumor regression with a PD-L1 antibody. Glutsch et al. [113] in their paper report that 3 out of 5 avelumab-refractory patients responded to the combination of ipilimumab plus nivolumab after failure of avelumab-based therapy, experiencing no grade 3 or 4 AEs.

\section{Conclusions}

Immunotherapy, at this time, finds its precise place in the treatment of SCLC and MCC. In these two forms of aggressive and high-grade NENs, ICIs have obtained important and encouraging results, which in the future will still be implemented by the ongoing trials regarding combined treatments among different ICIs and other molecules.

On the contrary, with regard to the well-differentiated forms of NENs, the results obtained so far have been disappointing and, unfortunately, immunotherapy cannot be considered a promising therapy at the moment. ICIs have no impact on clinical practice in the treatment of differentiated NENs and do not yet have a precise role in the therapeutic sequence of these tumors.

Considering the low TMB and the scarce tumor-infiltrating lymphocytes that characterize NETs, these results, obtained so far, are not surprising. This picture does not mean that in the future a better outcome cannot be obtained in patients with NETs as well. However, the most important aspect will be to study strategies that can make NETs more susceptible to response to ICI and, thus, enhance the effectiveness of these treatments. Therefore, the combination of conventional therapy, target therapy and immunotherapy deserves attention and warrant to be explored [114]

A sequential chemotherapy, possibly inducing an increase in TMB and tested before immunotherapy, could be a hypothesis deserving more consideration. Radiotherapy or PRRT, that precedes the start of ICI and induces an inflammation at tumoral level, with an increase of tumor-infiltrating lymphocytes, could be another approach to explore. Equally essential will be the identification of biomarkers useful for selecting patients potentially responsive to this type of treatment. In fact, responses to immunotherapy were also reported in tumors presented with low PD-L1 expression and low TMB, underlying that a screening of patients based only on these two biomarkers could exclude potential responders to ICIs $[32,33]$.

Some intrinsic characteristics of NENs make difficult to study all these hypotheses, since the large heterogeneity of NENs in their biology and clinical behavior, and their relative rarity, combined with the habit of conducting studies on nonhomogeneous case-series by primitive site or grading, do not allow to obtain univocal and established data on this topic. Until we have clarified all these aspects, it will be difficult for ICI to revolutionize the therapeutic approach of welldifferentiated NENs. 
Funding Open access funding provided by Università degli Studi di Genova within the CRUI-CARE Agreement. The study was partially supported by funds of the Italian Ministry of Education, University and Research (PRIN 2017Z3N3YC).

\section{Declarations}

Conflict of interest The authors declare that there is no conflict of interest that could be perceived as prejudicing the impartiality of this review.

Open Access This article is licensed under a Creative Commons Attribution 4.0 International License, which permits use, sharing, adaptation, distribution and reproduction in any medium or format, as long as you give appropriate credit to the original author(s) and the source, provide a link to the Creative Commons licence, and indicate if changes were made. The images or other third party material in this article are included in the article's Creative Commons licence, unless indicated otherwise in a credit line to the material. If material is not included in the article's Creative Commons licence and your intended use is not permitted by statutory regulation or exceeds the permitted use, you will need to obtain permission directly from the copyright holder. To view a copy of this licence, visit http://creativecommons.org/licenses/by/4.0/.

\section{References}

1. Torre LA, Bray F, Siegel RL, Ferlay J, Lortet-Tieulent J, Jemal A. Global cancer statistics, 2012. CA Cancer J Clin. 2015;65(2):87-108.

2. DeVita VT, Chu E. A history of cancer chemotherapy. Cancer Res. 2008;68(21):8643-53.

3. Lee YT, Tan YJ, Oon CE. Molecular targeted therapy: Treating cancer with specificity. Eur J Pharmacol. 2018;5(834):188-96.

4. Helmy KY, Patel SA, Nahas GR, Rameshwar P. Cancer immunotherapy: accomplishments to date and future promise. Ther Deliv. 2013;4(10):1307-20.

5. Hanahan D, Weinberg RA. The hallmarks of cancer. Cell. 2000;100(1):57-70.

6. Hanahan D, Weinberg RA. Hallmarks of cancer: the next generation. Cell. 2011;144(5):646-74.

7. Leach DR, Krummel MF, Allison JP. Enhancement of antitumor immunity by CTLA-4 blockade. Science. 1996;271(5256):1734-6.

8. Dong H, Strome SE, Salomao DR, Tamura H, Hirano F, Flies $\mathrm{DB}$, et al. Tumor-associated B7-H1 promotes T-cell apoptosis: a potential mechanism of immune evasion. Nat Med. 2002;8(8):793-800.

9. Melero I, Berman DM, Aznar MA, Korman AJ, Pérez Gracia JL, Haanen J. Evolving synergistic combinations of targeted immunotherapies to combat cancer. Nat Rev Cancer. 2015;15(8):457-72.

10. Topalian SL, Drake CG, Pardoll DM. Immune checkpoint blockade: a common denominator approach to cancer therapy. Cancer Cell. 2015;27(4):450-61.

11. Mooradian MJ, Sullivan RJ. Immunomodulatory effects of current cancer treatment and the consequences for follow-up immunotherapeutics. Future Oncol. 2017;13(18):1649-63.

12. Milling L, Zhang Y, Irvine DJ. Delivering safer immunotherapies for cancer. Adv Drug Deliv Rev. 2017;15(114):79-101.

13. Wang J-J, Lei K-F, Han F. Tumor microenvironment: recent advances in various cancer treatments. Eur Rev Med Pharmacol Sci. 2018;22(12):3855-64.
14. Modlin IM, Oberg K, Chung DC, Jensen RT, de Herder WW, Thakker RV, et al. Gastroenteropancreatic neuroendocrine tumours. Lancet Oncol. 2008;9(1):61-72.

15. Alexandraki KI, Tsoli M, Kyriakopoulos G, Angelousi A, Nikolopoulos G, Kolomodi D, et al. Current concepts in the diagnosis and management of neuroendocrine neoplasms of unknown primary origin. Minerva Endocrinol. 2019;44(4):378-86.

16. Klimstra DS, Kloppel G, La Rosa S, Rindi G. Classification of neuroendocrine neoplasms of the digestive system. In WHO Classification of Tumors of the Digestive System, 5th ed.; IARC Press: Lyon, France, 2019; Volume 1

17. Lloyd RV, Osamura RY, Klöppel GRJ. WHO Classification of Tumours of Endocrine Organs. 4th ed. Geneva, Switzerland: WHO; 2017.

18. Pavel M, O’Toole D, Costa F, Capdevila J, Gross D, Kianmanesh $\mathrm{R}$, et al. ENETS Consensus Guidelines Update for the Management of Distant Metastatic Disease of Intestinal, Pancreatic, Bronchial Neuroendocrine Neoplasms (NEN) and NEN of Unknown Primary Site. Neuroendocrinology. 2016;103(2):172-85.

19. Femia D, Prinzi N, Anichini A, Mortarini R, Nichetti F, Corti F, et al. Treatment of Advanced Merkel Cell Carcinoma: Current Therapeutic Options and Novel Immunotherapy Approaches. Target Oncol. 2018;13(5):567-82.

20. Fanciulli G, Di Molfetta S, Dotto A, Florio T, Feola T, Rubino M, et al. Emerging Therapies in Pheochromocytoma and Paraganglioma: Immune Checkpoint Inhibitors in the Starting Blocks. J Clin Med. 2020 Dec 29;10(1).

21. Yao JC, Hassan M, Phan A, Dagohoy C, Leary C, Mares JE, et al. One hundred years after "carcinoid": epidemiology of and prognostic factors for neuroendocrine tumors in 35,825 cases in the United States. J Clin Oncol. 2008;26(18):3063-72.

22. SEER Cancer Statistics Review 1975-2004 - Previous Version - SEER Cancer Statistics [Internet]. SEER. [cited $2021 \mathrm{Feb}$ 10]. Available from: https://seer.cancer.gov/archive/csr/1975_2004/index.html

23. Cives M, Strosberg JR. Gastroenteropancreatic Neuroendocrine Tumors. CA Cancer J Clin. 2018;68(6):471-87.

24. NCCN Clinical Practice Guidelines in Oncology. Neuroendocrine and Adrenal tumors. Version 1.2020. [cited 2021 Feb 10]. Available from: https://www.nccn.org/professionals/physician_ gls/default.aspx

25. Strosberg J, El-Haddad G, Wolin E, Hendifar A, Yao J, Chasen $\mathrm{B}$, et al. Phase 3 Trial of 177Lu-Dotatate for Midgut Neuroendocrine Tumors. N Engl J Med. 2017;376(2):125-35.

26. Mitry E, Baudin E, Ducreux M, Sabourin JC, Rufié P, Aparicio T, et al. Treatment of poorly differentiated neuroendocrine tumours with etoposide and cisplatin. Br J Cancer. 1999;81(8):1351-5.

27. Sorbye H, Welin S, Langer SW, Vestermark LW, Holt N, Osterlund $\mathrm{P}$, et al. Predictive and prognostic factors for treatment and survival in 305 patients with advanced gastrointestinal neuroendocrine carcinoma (WHO G3): the NORDIC NEC study. Ann Oncol. 2013;24(1):152-60.

28. Garcia-Carbonero R, Sorbye H, Baudin E, Raymond E, Wiedenmann B, Niederle B, et al. ENETS Consensus Guidelines for High-Grade Gastroenteropancreatic Neuroendocrine Tumors and Neuroendocrine Carcinomas. Neuroendocrinology. 2016;103(2):186-94.

29. Al-Toubah T, Cives M, Strosberg J. Novel immunotherapy strategies for treatment of neuroendocrine neoplasms. Transl Gastroenterol Hepatol. 2020;5:54.

30. Cives M, Pelle' E, Strosberg J. Emerging Treatment Options for Gastroenteropancreatic Neuroendocrine Tumors. J Clin Med [Internet]. 2020 Nov 13 [cited 2021 Feb 10];9(11). Available from: https://www.ncbi.nlm.nih.gov/pmc/articles/PMC7696369/

31. Rizvi H, Sanchez-Vega F, La K, Chatila W, Jonsson P, Halpenny D, et al. Molecular Determinants of Response to 
Anti-Programmed Cell Death (PD)-1 and Anti-Programmed Death-Ligand 1 (PD-L1) Blockade in Patients With Non-SmallCell Lung Cancer Profiled With Targeted Next-Generation Sequencing. J Clin Oncol. 2018;36(7):633-41.

32. Wallis CJD, Lawson K, Butaney M, Satkunasivam R, Parikh J, Freedland SJ, et al. Association between PD-L1 status and immune checkpoint inhibitor response in advanced malignancies: a systematic review and meta-analysis of overall survival data. Jpn J Clin Oncol. 2020;50(7):800-9.

33. Samstein RM, Lee C-H, Shoushtari AN, Hellmann MD, Shen $\mathrm{R}$, Janjigian $\mathrm{YY}$, et al. Tumor mutational load predicts survival after immunotherapy across multiple cancer types. Nat Genet. $2019 \mathrm{Feb} ; 51(2): 202-6.4$.

34. Cives M, Pelle' E, Quaresmini D, Rizzo FM, Tucci M, Silvestris F. The Tumor Microenvironment in Neuroendocrine Tumors: Biology and Therapeutic Implications. Neuroendocrinology. 2019;109(2):83-99.

35. Cives M, Strosberg J, Al Diffalha S, Coppola D. Analysis of the immune landscape of small bowel neuroendocrine tumors. Endocr Relat Cancer. 2019;26(1):119-30.

36. Da Silva A, Bowden M, Zhang S, Masugi Y, Thorner AR, Herbert ZT, et al. Characterization of the Neuroendocrine Tumor Immune Microenvironment. Pancreas. 2018;47(9):1123-9.

37. Katz SC, Donkor C, Glasgow K, Pillarisetty VG, Gönen M, Espat NJ, et al. T cell infiltrate and outcome following resection of intermediate-grade primary neuroendocrine tumours and liver metastases. HPB (Oxford). 2010;12(10):674-83.

38. Vitale G, Carra S, Ferraù F, Guadagno E, Faggiano A, Colao A, et al. Gastroenteropancreatic neuroendocrine neoplasms and inflammation: A complex cross-talk with relevant clinical implications. Crit Rev Oncol Hematol. 2020;146:102840.

39. Sampedro-Núñez M, Serrano-Somavilla A, Adrados M, Cameselle-Teijeiro JM, Blanco-Carrera C, Cabezas-Agricola $\mathrm{JM}$, et al. Analysis of expression of the PD-1/PD-L1 immune checkpoint system and its prognostic impact in gastroenteropancreatic neuroendocrine tumors. Sci Rep. 2018;8(1):17812.

40. Bösch F, Brüwer K, Altendorf-Hofmann A, Auernhammer CJ, Spitzweg C, Westphalen CB, et al. Immune checkpoint markers in gastroenteropancreatic neuroendocrine neoplasia. Endocr Relat Cancer. 2019;26(3):293-301.

41. Kim ST, Ha SY, Lee S, Ahn S, Lee J, Park SH, et al. The Impact of PD-L1 Expression in Patients with Metastatic GEPNETs. J Cancer. 2016;7(5):484-9.

42. Cavalcanti E, Armentano R, Valentini AM, Chieppa M, Caruso ML. Role of PD-L1 expression as a biomarker for GEP neuroendocrine neoplasm grading. Cell Death Dis. 2017;8(8):e3004.

43. Ali AS, Langer SW, Federspiel B, Hjortland GO, Grønbæk H, Ladekarl M, et al. PD-L1 expression in gastroenteropancreatic neuroendocrine neoplasms grade 3. PLoS One. 2020;15(12):e0243900.

44. Salem ME, Puccini A, Grothey A, Raghavan D, Goldberg RM, Xiu J, et al. Landscape of Tumor Mutation Load, Mismatch Repair Deficiency, and PD-L1 Expression in a Large Patient Cohort of Gastrointestinal Cancers. Mol Cancer Res. 2018;16(5):805-12.

45. Ott PA, Bang Y-J, Piha-Paul SA, Razak ARA, Bennouna J, Soria J-C, et al. T-Cell-Inflamed Gene-Expression Profile, Programmed Death Ligand 1 Expression, and Tumor Mutational Burden Predict Efficacy in Patients Treated With Pembrolizumab Across 20 Cancers: KEYNOTE-028. J Clin Oncol. 2019;37(4):318-27.

46. Strosberg J, Mizuno N, Doi T, Grande E, Delord J-P, ShapiraFrommer R, et al. Efficacy and Safety of Pembrolizumab in Previously Treated Advanced Neuroendocrine Tumors: Results From the Phase II KEYNOTE-158 Study. Clin Cancer Res. 2020;26(9):2124-30.
47. Yao JC, Strosberg J, Fazio N, Pavel ME, Bergsland E, Ruszniewski $\mathrm{P}$, et al.: Spartalizumab in metastatic, well/poorlydifferentiated neuroendocrine neoplasms. Endocr Relat Cancer. (2021).

48. Lu M, Zhang P, Zhang Y, Li Z, Gong J, Li J, et al. Efficacy, Safety, and Biomarkers of Toripalimab in Patients with Recurrent or Metastatic Neuroendocrine Neoplasms: A Multiple-Center Phase Ib Trial. Clin Cancer Res. 2020;26(10):2337-45.

49. Patel SP, Othus M, Chae YK, Giles FJ, Hansel DE, Singh PP, et al. A Phase II Basket Trial of Dual Anti-CTLA-4 and AntiPD-1 Blockade in Rare Tumors (DART SWOG 1609) in Patients with Nonpancreatic Neuroendocrine Tumors. Clin Cancer Res. 2020;26(10):2290-6.

50. Klein O, Kee D, Markman B, Michael M, Underhill C, Carlino $\mathrm{MS}$, et al. Immunotherapy of Ipilimumab and Nivolumab in Patients with Advanced Neuroendocrine Tumors: A Subgroup Analysis of the CA209-538 Clinical Trial for Rare Cancers. Clin Cancer Res. 2020;26(17):4454-9.

51. Fottner C, Apostolidis L, Ferrata M, Krug S, Michl P, Schad A, et al. A phase II, open label, multicenter trial of avelumab in patients with advanced, metastatic high-grade neuroendocrine carcinomas NEC G3 (WHO 2010) progressive after first-line chemotherapy (AVENEC). JCO. 2019 May 20;37(15_suppl):4103-4103.

52. Morse M, Halperin DM, Uronis HE, Hsu DS, Hurwitz H, Bolch E, et al. Phase Ib/II study of pembrolizumab with lanreotide depot for advanced, progressive gastroenteropancreatic neuroendocrine tumors (PLANET). JCO. 2021 Jan 20;39(3_suppl):369-369.

53. Owen DH, Wei L, Goyal A, Zhou Y, Suffren S-A, Jacob R, et al. CLO20-054: A Phase 2 Trial of Nivolumab and Temozolomide in Advanced Neuroendocrine Tumors (NETs): Interim Efficacy Analysis. Journal of the National Comprehensive Cancer Network. 2020 Mar 20;18(3.5):CLO20-CLO20-054.

54. Uboha NV, Milhem MM, Kovacs C, Amin A, Magley A, Purkayastha DD, et al. Phase II study of spartalizumab (PDR001) and LAG525 in advanced solid tumors and hematologic malignancies. JCO. 2019 May 20;37(15_suppl):2553-2553.

55. Halperin DM, Liu S, Dasari A, Fogelman DR, Bhosale P, Mahvash A, et al. A phase II trial of atezolizumab and bevacizumab in patients with advanced, progressive neuroendocrine tumors (NETs). JCO. 2020 Feb 1;38(4_suppl):619-619.

56. Mulvey C, Raj NP, Chan JA, Aggarwal RR, Cinar P, Hope TA, et al. Phase II study of pembrolizumab-based therapy in previously treated extrapulmonary poorly differentiated neuroendocrine carcinomas: Results of Part A (pembrolizumab alone). JCO. 2019 Jan 29;37(4_suppl):363-363.

57. Capdevila J, Teule A, López C, García-Carbonero R, Benavent M, Custodio A, et al. 11570 A multi-cohort phase II study of durvalumab plus tremelimumab for the treatment of patients (pts) with advanced neuroendocrine neoplasms (NENs) of gastroenteropancreatic or lung origin: The DUNE trial (GETNE 1601). Ann Oncol. 2020;1(31):S770-1.

58. Travis WD, Brambilla E, Nicholson AG, Yatabe Y, Austin JHM, Beasley MB, et al. The 2015 World Health Organization Classification of Lung Tumors: Impact of Genetic, Clinical and Radiologic Advances Since the 2004 Classification. J Thorac Oncol. 2015;10(9):1243-60.

59. Peri M, Fazio N. Clinical Evaluation of Everolimus in the Treatment of Neuroendocrine Tumors of the Lung: Patient Selection and Special Considerations. A Systematic and Critical Review of the Literature. Lung Cancer (Auckl). (2020) 8;11:41-52.

60. Brighi N, Lamberti G, Manuzzi L, Maggio I, Campana D. Therapeutic options in lung neuroendocrine tumors: between established concepts and new hopes. Anticancer Drugs. 2019;30(7):e0784. 
61. Vesterinen T, Kuopio T, Ahtiainen M, Knuuttila A, Mustonen H, Salmenkivi K, et al. PD-1 and PD-L1 expression in pulmonary carcinoid tumors and their association to tumor spread. Endocr Connect. 2019;8(8):1168-75.

62. Nestor J, Barnaby K, Esposito M, Seetharamu N. Treatment of atypical pulmonary carcinoid with combination ipilimumab and nivolumab. BMJ Case Rep. 2019 Nov 28;12(11).

63. Mauclet C, Duplaquet F, Pirard L, Rondelet B, Dupont M, PopStanciu C, et al. Complete tumor response of a locally advanced lung large-cell neuroendocrine carcinoma after palliative thoracic radiotherapy and immunotherapy with nivolumab. Lung Cancer. 2019; 128:53-6.

64. Levra MG, Mazieres J, Valette CA, Molinier O, Planchard D, Frappat V, et al. P1.07-012 Efficacy of Immune Checkpoint Inhibitors in Large Cell Neuroendocrine Lung Cancer: Results from a French Retrospective Cohort: Topic: Drug Treatment Alone and in Combination with Radiotherapy. J Thorac Oncol. 2017 Jan 1;12(1):S702-3.

65. Maggio I, Manuzzi L, Lamberti G, Ricci AD, Tober N, Campana D. Landscape and Future Perspectives of Immunotherapy in Neuroendocrine Neoplasia. Cancers (Basel). 2020 Mar 30;12(4).

66. Früh M, De Ruysscher D, Popat S, Crinò L, Peters S, Felip E, et al. Small-cell lung cancer (SCLC): ESMO Clinical Practice Guidelines for diagnosis, treatment and follow-up. Ann Oncol. 2013 Oct;24 Suppl 6:vi99-105.

67. Sabari JK, Lok BH, Laird JH, Poirier JT, Rudin CM. Unravelling the biology of SCLC: implications for therapy. Nat Rev Clin Oncol. 2017;14(9):549-61.

68. Horn L, Mansfield AS, Szczęsna A, Havel L, Krzakowski M, Hochmair MJ, et al. First-Line Atezolizumab plus Chemotherapy in Extensive-Stage Small-Cell Lung Cancer. N Engl J Med. 2018;379(23):2220-9.

69. Pujol J-L, Greillier L, Audigier-Valette C, Moro-Sibilot D, Uwer L, Hureaux J, et al. A Randomized Non-Comparative Phase II Study of Anti-Programmed Cell Death-Ligand 1 Atezolizumab or Chemotherapy as Second-Line Therapy in Patients With Small Cell Lung Cancer: Results From the IFCT-1603 Trial. J Thorac Oncol. 2019;14(5):903-13.

70. Gandhi L, Rodríguez-Abreu D, Gadgeel S, Esteban E, Felip E, De Angelis F, et al. Pembrolizumab plus Chemotherapy in Metastatic Non-Small-Cell Lung Cancer. N Engl J Med. 2018;378(22):2078-92.

71. Paz-Ares L, Dvorkin M, Chen Y, Reinmuth N, Hotta K, Trukhin $\mathrm{D}$, et al. Durvalumab plus platinum-etoposide versus platinumetoposide in first-line treatment of extensive-stage small-cell lung cancer (CASPIAN): a randomised, controlled, open-label, phase 3 trial. Lancet. 2019;394(10212):1929-39.

72. Reck M, Luft A, Szczesna A, Havel L, Kim S-W, Akerley W, et al. Phase III Randomized Trial of Ipilimumab Plus Etoposide and Platinum Versus Placebo Plus Etoposide and Platinum in Extensive-Stage Small-Cell Lung Cancer. J Clin Oncol. 2016;34(31):3740-8.

73. Owonikoko TK, Kim HR, Govindan R, Ready N, Reck M, Peters S, et al. Nivolumab (nivo) plus ipilimumab (ipi), nivo, or placebo (pbo) as maintenance therapy in patients (pts) with extensive disease small cell lung cancer (ED-SCLC) after first-line (1L) platinum-based chemotherapy (chemo): Results from the double-blind, randomized phase III CheckMate 451 study. Ann Oncol. 2019 Apr 1;30:ii77.

74. Spigel DR, Vicente D, Ciuleanu TE, Gettinger S, Peters S, Horn L, et al. Second-Line Nivolumab in Relapsed Small-Cell Lung Cancer: CheckMate 331. Ann Oncol. 2021 Feb 1;

75. Antonia SJ, López-Martin JA, Bendell J, Ott PA, Taylor M, Eder JP, et al. Nivolumab alone and nivolumab plus ipilimumab in recurrent small-cell lung cancer (CheckMate 032): a multicentre, open-label, phase 1/2 trial. Lancet Oncol. 2016;17(7):883-95.
76. Ready N, Farago AF, de Braud F, Atmaca A, Hellmann MD, Schneider JG, et al. Third-Line Nivolumab Monotherapy in Recurrent SCLC: CheckMate 032. J Thorac Oncol. 2019;14(2):237-44.

77. Chung HC, Lopez-Martin JA, Kao SC-H, Miller WH, Ros W, Gao B, et al. Phase 2 study of pembrolizumab in advanced smallcell lung cancer (SCLC): KEYNOTE-158. JCO. 2018 May 20;36(15_suppl):8506-8506.

78. Chung HC, Piha-Paul SA, Lopez-Martin J, Schellens JHM, Kao S, Miller WH, et al. Pembrolizumab After Two or More Lines of Previous Therapy in Patients With Recurrent or Metastatic SCLC: Results From the KEYNOTE-028 and KEYNOTE-158 Studies. J Thorac Oncol. 2020;15(4):618-27.

79. Toker C. Trabecular carcinoma of the skin. Arch Dermatol. 1972;105(1):107-10.

80. Tilling T, Moll I. Which are the cells of origin in merkel cell carcinoma? J Skin Cancer. 2012;2012:680410.

81. Feng H, Shuda M, Chang Y, Moore PS. Clonal integration of a polyomavirus in human Merkel cell carcinoma. Science. 2008;319(5866):1096-100.

82. Hall ET, Fernandez-Lopez E, Silk AW, Dummer R, Bhatia S. Immunologic Characteristics of Nonmelanoma Skin Cancers: Implications for Immunotherapy. Am Soc Clin Oncol Educ Book. 2020;40:1-10.

83. Hasan S, Liu L, Triplet J, Li Z, Mansur D. The role of postoperative radiation and chemoradiation in merkel cell carcinoma: a systematic review of the literature. Front Oncol. 2013;3:276.

84. Engels EA, Frisch M, Goedert JJ, Biggar RJ, Miller RW. Merkel cell carcinoma and HIV infection. Lancet. 2002;359(9305):497-8.

85. Agelli M, Clegg LX, Becker JC, Rollison DE. The etiology and epidemiology of merkel cell carcinoma. Curr Probl Cancer. 2010;34(1):14-37.

86. Paulson KG, Iyer JG, Blom A, Warton EM, Sokil M, Yelistratova L, et al. Systemic Immune Suppression Predicts Diminished Merkel Cell Carcinoma-Specific Survival Independent of Stage. J Investig Dermatol. 2013;133(3):642-6.

87. Merkel UH, Carcinoma C. An Update and Immunotherapy Front Oncol. 2018;8:48.

88. Vandeven N, Lewis CW, Makarov V, Riaz N, Paulson KG, Hippe D, et al. Merkel Cell Carcinoma Patients Presenting Without a Primary Lesion Have Elevated Markers of Immunity, Higher Tumor Mutation Burden, and Improved Survival. Clin Cancer Res. 2018;24(4):963-71.

89. Lyngaa R, Pedersen NW, Schrama D, Thrue CA, Ibrani D, Met $\mathrm{O}$, et al. T-cell responses to oncogenic merkel cell polyomavirus proteins distinguish patients with merkel cell carcinoma from healthy donors. Clin Cancer Res. 2014;20(7):1768-78.

90. Houben R, Shuda M, Weinkam R, Schrama D, Feng H, Chang $\mathrm{Y}$, et al. Merkel cell polyomavirus-infected Merkel cell carcinoma cells require expression of viral T antigens. J Virol. 2010;84(14):7064-72.

91. Harms PW, Vats P, Verhaegen ME, Robinson DR, Wu Y-M, Dhanasekaran SM, et al. The Distinctive Mutational Spectra of Polyomavirus-Negative Merkel Cell Carcinoma. Cancer Res. 2015;75(18):3720-7.

92. Paulson KG, Iyer JG, Tegeder AR, Thibodeau R, Schelter J, Koba S, et al. Transcriptome-Wide Studies of Merkel Cell Carcinoma and Validation of Intratumoral CD8+ Lymphocyte Invasion As an Independent Predictor of Survival. J Clin Oncol. 2011;29(12):1539-46.

93. Wong SQ, Waldeck K, Vergara IA, Schröder J, Madore J, Wilmott JS, et al. UV-Associated Mutations Underlie the Etiology of MCV-Negative Merkel Cell Carcinomas. Cancer Res. 2015;75(24):5228-34.

94. Schadendorf D, Nghiem P, Bhatia S, Hauschild A, Saiag P, Mahnke L, et al. Immune evasion mechanisms and immune 
checkpoint inhibition in advanced merkel cell carcinoma. Oncoimmunology. 2017;6(10):e1338237.

95. FDA highlights of prescribing information. https://www.accessdata. fda.gov/drugsatfda_docs/label/2019/761049s006lbl.pdf [accessed 2021 Feb 10].

96. FDA highlights of prescribing information. https://www.accessdata. fda.gov/drugsatfda_docs/label/2020/125514s066lbl.pdf [accessed 2021 Feb 10].

97. Collins JM, Gulley JL. Product review: avelumab, an anti-PD-L1 antibody. Hum Vaccin Immunother. 2019;15(4):891-908.

98. Kelly K, Infante JR, Taylor MH, Patel MR, Wong DJ, Iannotti N, et al. Safety profile of avelumab in patients with advanced solid tumors: A pooled analysis of data from the phase 1 JAVELIN solid tumor and phase 2 JAVELIN Merkel 200 clinical trials. Cancer. 2018;124(9):2010-7.

99. Kaufman HL, Russell J, Hamid O, Bhatia S, Terheyden P, D’Angelo $\mathrm{SP}$, et al. Avelumab in patients with chemotherapy-refractory metastatic Merkel cell carcinoma: a multicentre, single-group, openlabel, phase 2 trial. Lancet Oncol. 2016;17(10):1374-85.

100. Kaufman HL, Russell JS, Hamid O, Bhatia S, Terheyden P, D'Angelo SP, et al. Updated efficacy of avelumab in patients with previously treated metastatic Merkel cell carcinoma after $\geq 1$ year of follow-up: JAVELIN Merkel 200, a phase 2 clinical trial. J Immunother Cancer. 2018;6(1):7.

101. D’Angelo SP, Russell J, Lebbé C, Chmielowski B, Gambichler T, Grob J-J, et al. Efficacy and Safety of First-line Avelumab Treatment in Patients With Stage IV Metastatic Merkel Cell Carcinoma: A Preplanned Interim Analysis of a Clinical Trial. JAMA Oncol. 2018;4(9):e180077.

102. Walker JW, Lebbé C, Grignani G, Nathan P, Dirix L, Fenig E, et al. Efficacy and safety of avelumab treatment in patients with metastatic Merkel cell carcinoma: experience from a global expanded access program. J Immunother Cancer [Internet]. 2020 Apr 8 [cited $2021 \mathrm{Feb} 10] ; 8(1)$. Available from: https://www. ncbi.nlm.nih.gov/pmc/articles/PMC7252959/

103. Nghiem P, Bhatia S, Lipson EJ, Sharfman WH, Kudchadkar RR, Brohl AS, et al. Durable Tumor Regression and Overall Survival in Patients With Advanced Merkel Cell Carcinoma Receiving Pembrolizumab as First-Line Therapy. JCO. 2019;37(9):693-702.

104. Nghiem PT, Bhatia S, Lipson EJ, Kudchadkar RR, Miller NJ, Annamalai L, et al. PD-1 Blockade with Pembrolizumab in Advanced Merkel-Cell Carcinoma. N Engl J Med. 2016; 374(26):2542-52.

105. Topalian SL, Bhatia S, Hollebecque A, Awada A, Boer JPD, Kudchadkar RR, et al. Abstract CT074: Non-comparative, openlabel, multiple cohort, phase $1 / 2$ study to evaluate nivolumab
(NIVO) in patients with virus-associated tumors (CheckMate 358): Efficacy and safety in Merkel cell carcinoma (MCC). Cancer Res. 2017 Jul 1;77(13 Supplement):CT074-CT074.

106. Topalian SL, Bhatia S, Amin A, Kudchadkar RR, Sharfman WH, Lebbé C, et al. Neoadjuvant Nivolumab for Patients With Resectable Merkel Cell Carcinoma in the CheckMate 358 Trial. J Clin Oncol. 2020;38(22):2476-87.

107. Bichakjian CK, Olencki T, Aasi SZ, Alam M, Andersen JS, Blitzblau R, et al. Merkel Cell Carcinoma, Version 1.2018, NCCN Clinical Practice Guidelines in Oncology. J Natl Compr Canc Netw. 2018 Jun;16(6):742-74.

108. Knepper TC, Montesion M, Russell JS, Sokol ES, Frampton GM, Miller VA, et al. The Genomic Landscape of Merkel Cell Carcinoma and Clinicogenomic Biomarkers of Response to Immune Checkpoint Inhibitor Therapy. Clin Cancer Res. 2019;25(19):5961-71.

109. Spassova I, Ugurel S, Terheyden P, Sucker A, Hassel JC, Ritter C, et al. Predominance of Central Memory T Cells with High T-Cell Receptor Repertoire Diversity is Associated with Response to PD-1/PD-L1 Inhibition in Merkel Cell Carcinoma. Clin Cancer Res. 2020;26(9):2257-66.

110. Bystrup Boyles T, Schødt M, Hendel HW, Krarup-Hansen A, Junker N. Pembrolizumab as first line treatment of Merkel cell carcinoma patients - a case series of patients with various comorbidities. Acta Oncol. 2020;59(7):793-6.

111. Levy S, Aarts MJB, Eskens FALM, Keymeulen KBMI, Been LB, Grünhagen D, et al. Avelumab for advanced Merkel cell carcinoma in the Netherlands: a real-world cohort. J Immunother Cancer. 2020 Sep;8(2).

112. LoPiccolo J, Schollenberger MD, Dakhil S, Rosner S, Ali O, Sharfman WH, et al. Rescue therapy for patients with anti-PD1-refractory Merkel cell carcinoma: a multicenter, retrospective case series. J Immunother Cancer. 2019;7(1):170.

113. Glutsch V, Kneitz H, Gesierich A, Goebeler M, Haferkamp $\mathrm{S}$, Becker JC, et al. Activity of ipilimumab plus nivolumab in avelumab-refractory Merkel cell carcinoma. Cancer Immunol Immunother. 2021 Jan 13;

114. Gotwals P, Cameron S, Cipolletta D, Cremasco V, Crystal A, Hewes B, et al. Prospects for combining targeted and conventional cancer therapy with immunotherapy. Nat Rev Cancer. 2017;17(5):286-301.

Publisher's Note Springer Nature remains neutral with regard to jurisdictional claims in published maps and institutional affiliations. 